\title{
Analysis of Two-Layered Random Interfaces for Two Dimensional Widom-Rowlinson's Model
}

\author{
Jun Wang, Jiguang Shao, and Bingtuan Wang \\ Department of Mathematics, Key Laboratory of Communication and Information System, \\ Beijing Jiaotong University, Beijing 100044, China \\ Correspondence should be addressed to Jun Wang, wangjun@center.njtu.edu.cn
}

Received 21 May 2011; Revised 31 August 2011; Accepted 12 September 2011

Academic Editor: Dumitru Baleanu

Copyright (C) 2011 Jun Wang et al. This is an open access article distributed under the Creative Commons Attribution License, which permits unrestricted use, distribution, and reproduction in any medium, provided the original work is properly cited.

\begin{abstract}
The statistical behaviors of two-layered random-phase interfaces in two-dimensional WidomRowlinson's model are investigated. The phase interfaces separate two coexisting phases of the lattice Widom-Rowlinson model; when the chemical potential $\mu$ of the model is large enough, the convergence of the probability distributions which describe the fluctuations of the phase interfaces is studied. In this paper, the backbones of interfaces are introduced in the model, and the corresponding polymer chains and cluster expansions are developed and analyzed for the polymer weights. And the existence of the free energy for two-layered random-phase interfaces of the two-dimensional Widom-Rowlinson model is given.
\end{abstract}

\section{Introduction}

We investigate the statistical behaviors of random interfaces between the two coexisting phases of the Widom-Rowlinson model (W-R model) when the chemical potential $\mu$ is large enough; especially we consider the two-layered interfaces behaviors of the model in this paper. The lattice system interfaces in two dimensions are known to fluctuate widely, for example, see [1-4] for the W-R model and [5-13] for the Ising spin system. There are two types of particles (either $A$ or $B$ ) in the lattice W-R system, and there is a strong repulsive interaction between particles of the different types. Namely, a $B$ particle cannot occupy a site within distance $\sqrt{2}$ from a site where an $A$ particle has occupied and vice versa. This means that different types of particles are separated by the empty sites. In [2], under some special conditions for the interfaces (with specified values of the area enclosed below interfaces and the height difference of two endpoints) and the chemical potential $\mu$ large enough, it shows the weak convergence of the probability distributions (which describe the fluctuations of such interfaces) to certain conditional Gaussian distribution. According to the dynamic system of the 
$\mathrm{W}-\mathrm{R}$ model and the results of [2], the thickness of the random interface (or the intermediate "belt") between the two coexisting phases of the model is expected to become thinner as $\mu$ becomes larger, so we believe that the interfaces of the W-R model behave like those of the Ising model to some extent. We are also interested in the fluctuation behaviors of two or more random interfaces that one interface lies above the other one, such model presents the coexistence of three or more phases, which is the multilayer interacting interface model. In the present paper, the two-layered lattice $\mathrm{W}-\mathrm{R}$ model is considered, and the convergence of the probability distributions which describe the fluctuations of two-layered random interfaces is exhibited.

The interface behavior of the lattice W-R model has close relation with the wetting phenomenon of the well-known Potts model, for example, see [14-17]. Wetting may occur when three or more phases coexist; it consists of the appearance of a thick (macroscopic) layer of the $C$ phase at an interface between the $A$ and the $B$ phases. Much research work has been devoted to the study of the wetting behavior for the $q$-state Potts model. Derrida and Schick [16] show that the interface is wetted by the disordered phase as the transition is approached by the mean field approximation. According to the method of low-temperature expansions, Bricmont and Lebowitz [14] exhibit the wetting of the interface between two ordered phases by the disordered one with $q$ being large. At the transition point, De Coninck et al. [15] display the similar wetting behavior by the correlation inequalities, and Messager et al. [17] present an analysis of the order-disorder transition for large $q$ based on the theory of cluster expansion and surface tension of the Potts model. For the interface of the lattice W-R system, we think that the wetting phenomenon may appear when the positive chemical potential $\mu$ is small, which means that the layer of empty sites that separate $A$ and $B$ particles may have some thickness. Whereas the results of the present paper may imply that the interface of the W-R model will not be wetted for large parameter $\mu$, since we think that the layer between the two coexisting phases is expected to become thinner as $\mu$ becomes larger.

We consider the two-layered phase interfaces in the two-dimensional Widom-Rowlinson model on the rectangle $\Lambda_{L, M}$, where $\Lambda_{L, M}=[1, L-1] \times[-M, M] \subset \mathbb{Z}^{2}$. Suppose that the particles in $\Lambda_{L, M}$ are of two types and there is a strong repulsive interaction between particles of the different types. Let $\sigma$ denote a configuration on $\{-1,0,+1\}^{\Lambda_{L, M}}$, where $\sigma(x)=+1$ denotes that the site $x$ is occupied by an $A$ particle, $\sigma(x)=-1$ denotes that $x$ is occupied by a $B$ particle, and $\sigma(x)=0$ denotes that there is no particle at $x$. We say that a configuration $\sigma$ is feasible if $\sigma(x) \sigma(y) \geq 0$ for all pairs $x, y \in \mathbb{Z}^{2}$ with $|x-y| \leq \sqrt{2}$, where $|\cdot|$ is the Euclidean distance. Let $\Omega_{L, M}$ denote the set of all feasible configurations in $\Lambda_{L, M}$, so there is a finite diameter hardcore exclusion between $A$ particles and $B$ particles on $\Omega_{L, M}$. Next, the two-layer interface model is defined for satisfying the following three conditions.

(i) The Hamiltonian of the two-layered model is given by

$$
H_{L, M}(\sigma)=-\sum_{x \in \Lambda_{L, M}} \mu\left(\sigma(x)^{2}-1\right)
$$

for all $\sigma \in \Omega_{L, M}$, where $\mu(>0)$ denotes the chemical potential. 
(ii) Let $b_{1}=b_{1}(L)>0$, let $b_{2}=b_{2}(L)>0$, and assume that $M>b_{1}, M>b_{2}$. We define a boundary condition $\omega^{b}$ as

$$
\omega^{b}(x)=\omega^{b}\left(x^{1}, x^{2}\right)=\left\{\begin{aligned}
+1, & \text { if } x^{1}=0,\left|x^{2}\right|>b_{1} \text { and if } x^{1}=L,\left|x^{2}\right|>b_{2} \\
& \text { and if } 1 \leq x^{1} \leq L-1,\left|x^{2}\right|=M+1, \\
0, & \text { if } x^{1}=0,\left|x^{2}\right|=b_{1} \text { and if } x^{1}=L,\left|x^{2}\right|=b_{2} \\
-1, & \text { if } x^{1}=0,\left|x^{2}\right|<b_{1} \text { and if } x^{1}=L,\left|x^{2}\right|<b_{2}
\end{aligned}\right.
$$

for each $x=\left(x^{1}, x^{2}\right) \in \partial \Lambda_{L, M}=[0, L] \times[-M-1, M+1] \backslash \Lambda_{L, M}$.

(iii) Suppose that there is a connected " -1 " particles path from the left side of $[0, L] \times$ $[-M-1, M+1]$ to the right side.

The Hamiltonian of (i) is the same as that of the W-R model, and conditions (ii) and (iii) (here we suppose that $b_{1}(L)=c_{1} L, b_{2}(L)=c_{2} L$, for some $0<c_{1}, c_{2}<1$ ) ensure that we have the two-layered interface model. Let $\Omega_{L, M}^{b}\left(\subset \Omega_{L, M}\right)$ be the corresponding configuration space with the conditions (i)-(iii), such that the configuration $\sigma \times \omega^{b}$ is feasible, where $\sigma \times$ $\omega^{b}(x)=\sigma(x)$ for $x \in \Lambda_{L, M}$ and $\sigma \times \omega^{b}(x)=\omega^{b}(x)$ for $x \in \partial \Lambda_{L, M}$. For a fixed configuration $\sigma \in \Omega_{L, M}^{b}$ let $S^{0}(\sigma)$ denote the set of points in $\Lambda_{L, M}$ such that the configuration $\sigma$ takes 0 value. The connected components of $S^{0}(\sigma)$ are called contours; among these contours, there are two contours $\Gamma^{u}(\sigma)$ and $\Gamma^{l}(\sigma)$ which are defined as the interfaces of the model. $\Gamma^{u}(\sigma)$ is the upper interface with the starting point $\left(0, b_{1}\right)$ and the ending point $\left(L, b_{2}\right) ; \Gamma^{l}(\sigma)$ is the lower interface with the starting point $\left(0,-b_{1}\right)$ and the ending point $\left(L,-b_{2}\right)$. Let

$$
\mathcal{S}_{L, M}^{b, u}=\left\{\Gamma^{u}(\sigma) ; \sigma \in \Omega_{L, M}^{b}\right\}, \quad \mathcal{S}_{L, M}^{b, l}=\left\{\Gamma^{l}(\sigma) ; \sigma \in \Omega_{L, M}^{b}\right\}
$$

be the set of upper interfaces and the set of lower interfaces, respectively. The conditional Gibbs distribution on $\Omega_{L, M}^{b}$ with the boundary condition $\omega^{b}$ is given by

$$
P_{L, M}^{b}(\sigma)=\left(Z_{L, M}^{b}\right)^{-1} \exp \left\{-\mu\left|S^{0}(\sigma)\right|\right\}
$$

where $|S|$ denotes the cardinality of a set $S$, and $Z_{L, M}^{b}$ is the corresponding partition function.

\section{Backbones and Partition Functions}

The general theory of interfaces between the coexisting phases (which is based on its microscopic description) has been intensively studied, for example, see $[1,2,6,7,18]$. De Coninck et al. [18] introduce the SOS approximants for the Potts surface tensions and present a connection between the orientation-dependent surface tension of the Potts model and the corresponding surface tension of the SOS model. And they show that an SOS model is applied for the construction of the Potts crystal shapes. In this section, a similar approximation method is developed; that is, the interface of the W-R model can be approximated by its corresponding 
backbone. Next we introduce the definitions of the backbones $\pi\left(\Gamma^{u}\right)$ and $\pi\left(\Gamma^{l}\right)$ of interfaces $\Gamma^{u}$ and $\Gamma^{l}$ in the two-layered $\mathrm{W}-\mathrm{R}$ model and state the main results of the present paper. According to the above definitions of interfaces of the model, the interface of the two-layered model is an intermediate belt between the two coexisting phases, whereas the interface of the two-dimensional Ising model is an open polygon passing through the starting point to the ending point. This means that the methods and techniques of the partition function representation and the partition function cluster expansion, which are applied in analyzing the Ising model, cannot be directly used in analyzing the two-layered W-R model. In the present paper, we define a backbone $\pi\left(\Gamma^{u}\right)$ of $\Gamma^{u}$ to represent $\Gamma^{u}$; that is, among self-avoiding paths connecting the starting point $\left(0, b_{1}\right)$ with the ending point $\left(L, b_{2}\right)$ in $\Gamma^{u}$, we select a selfavoiding path (called backbone $\pi\left(\Gamma^{u}\right)$ ) by an "order" given in the following definition (6). Since the backbone $\pi\left(\Gamma^{u}\right)$ is an open polygon, this will help us to study the interfaces of the model. Now we define the set of self-avoiding paths in $\Gamma^{u}$ as

$$
\Pi_{\Gamma^{u}}=\text { The set of self-avoiding path in } \Gamma^{u} \text { connecting }\left(0, b_{1}\right) \text { with }\left(L, b_{2}\right) \text {. }
$$

Among these paths, we select a self-avoiding path according to the following order; the order is defined with preference among four directions:

$$
\text { up }>\text { down }>\text { right }>\text { left. }
$$

More precisely, let $\pi_{x}=\left\{x_{1}, \ldots, x_{n}\right\}$ and $\pi_{y}=\left\{y_{1}, \ldots, y_{m}\right\}$ be the two self-avoiding paths in $\Gamma$. Let $k=\min _{i \geq 1}\left\{i: x_{i} \neq y_{i}\right\}$ be the first number $i$ such that $x_{i} \neq y_{i}$. We define that $\pi_{x}>\pi_{y}$ if the direction of the ordered edge $\left\{x_{k-1}, x_{k}\right\}$ is preferred to the direction of the ordered edge $\left\{y_{k-1}, y_{k}\right\}$. Let $\pi\left(\Gamma^{u}\right)$ be the unique maximal element of $\Pi_{\Gamma^{u}}$ with respect to this order, and call $\pi\left(\Gamma^{u}\right)$ the backbone of $\Gamma^{u}$. Similarly, let $\pi\left(\Gamma^{l}\right)$ represent the backbone of $\Gamma^{l}$. In this paper, our study is mainly focused on the backbone of the phase separation belt.

Let $U_{1}, \ldots, U_{n}$ be the different connected subsets of $\Lambda_{L, M}$, we say that the subsets $\left\{U_{j}\right\}$ are compatible if they are connected components of the set $\cup_{1 \leq j \leq n} U_{j}$. We also say that $\left\{U_{j}\right\}$ are compatible with a connected set $G$ if $\left\{G, U_{j}\right\}$ are compatible for every $1 \leq j \leq n$. Next we define the hole of a connected set of $\mathbb{Z}^{2}$, we say that a set $D \subset \mathbb{Z}^{2}$ is $*$-connected if, for every $x, y \in D$, there exist a sequence $x=z_{0}, z_{1}, \ldots, z_{n}=y$ in $D$ such that $\left|z_{i}-z_{i-1}\right| \leq \sqrt{2}$ for every $1 \leq i \leq n$. A hole of a connected set $F \subset \mathbb{Z}^{2}$ is a finite $*$-connected component of $F^{c}=\mathbb{Z}^{2} \backslash F$. Since there may be some holes inside an interface of the W-R model (note that the interface of the Ising model has no such holes), there is a large difference of the partition function expansion between the W-R model and the Ising model. Therefore, the partition function $Z_{L, M}^{b}$ defined in (1.4) can be rewritten by the similar formulas in [1-3] as following:

$$
Z_{L, M}^{b}=\sum_{\Gamma^{u} \in \mathcal{S}_{L, M}^{b, u}, \Gamma^{l} \in \mathcal{S}_{L, M}^{b, l}} \sum_{\left\{U_{j}\right\}} 2^{N\left(\Gamma^{u}\right)+N\left(\Gamma^{l}\right)} e^{-\mu\left(\left|\Gamma^{u}\right|+\left|\Gamma^{l}\right|\right)} \prod_{j} 2^{N\left(U_{j}\right)} e^{-\mu\left|U_{j}\right|}
$$

where the second summation is taken over compatible families $\left\{U_{j}\right\}$, which are compatible with $\Gamma^{u} \cup \Gamma^{l},\left|\Gamma^{u}\right|$ is the number of points in $\Gamma^{u}$, and $N\left(\Gamma^{u}\right)$ is the number of holes in $\Gamma^{u}$, similar to $\Gamma^{l}, N\left(\Gamma^{l}\right)$, and $|U|, N(U)$. Then for some large $\mu_{0}>0$ and $\mu>\mu_{0}$, according to the theory of 
the cluster expansions (see [19]), we have

$$
\begin{aligned}
\frac{Z_{L, M}^{b}}{Z_{L, M}^{+}}= & \sum_{\Gamma^{u} \in \mathcal{S}_{L, M}^{b, u}, \Gamma^{l} \in \mathcal{S}_{L, M}^{b, l}} \\
& \times \exp \left\{-\mu\left(\left|\Gamma^{u}\right|+\left|\Gamma^{l}\right|\right)+\ln 2\left(N\left(\Gamma^{u}\right)+N\left(\Gamma^{l}\right)\right)-\sum_{\Lambda \subset \Lambda_{L, M}: \Lambda i\left(\Gamma^{u} \cup \Gamma^{l}\right)} \Phi(\Lambda)\right\},
\end{aligned}
$$

where $Z_{L, M}^{+}$is the partition function with the plus boundary condition, $\Lambda i\left(\Gamma^{u} \cup \Gamma^{l}\right)$ denotes that the set $\Lambda$ is incompatible with the interfaces $\Gamma^{u} \cup \Gamma^{l}$, and $\Phi(\Lambda)$ is a translation invariant function, which satisfies the following estimate:

$$
\sum_{\Lambda ; \Lambda \ni 0}|\Phi(\Lambda)| e^{\left(\mu-\mu_{0}\right)|\Lambda|}<1 .
$$

Moreover, if $\mu$ is sufficiently large, we have

$$
\begin{aligned}
\lim _{M \rightarrow \infty} \frac{Z_{L, M}^{b}}{Z_{L, M}^{+}}= & \sum_{\Gamma^{u} \in \mathcal{S}_{L^{b}, u}^{b} \Gamma^{l} \in \mathcal{S}_{L}^{b, l}} \\
& \times \exp \left\{-\mu\left(\left|\Gamma^{u}\right|+\left|\Gamma^{l}\right|\right)+\ln 2\left(N\left(\Gamma^{u}\right)+N\left(\Gamma^{l}\right)\right)-\sum_{\Lambda \subset \Lambda_{L, \infty}: \wedge i\left(\Gamma^{u} \cup \Gamma^{l}\right)} \Phi(\Lambda)\right\},
\end{aligned}
$$

where $\Lambda_{L, \infty}=[1, L-1] \times(-\infty, \infty) \cap \mathbb{Z}^{2}$ and

$$
\mathcal{S}_{L}^{b, u}=\cup_{M>0} \mathcal{S}_{L, M}^{b, u}, \quad \mathcal{S}_{L}^{b, l}=\cup_{M>0} \mathcal{S}_{L, M}^{b, l}
$$

Let

$$
W\left(\Gamma^{u}, \Gamma^{l}\right)=\exp \left\{-\mu\left(\left|\Gamma^{u}\right|+\left|\Gamma^{l}\right|\right)+\ln 2\left(N\left(\Gamma^{u}\right)+N\left(\Gamma^{l}\right)\right)-\sum_{\Lambda \subset \Lambda_{L, \infty}: \Lambda i\left(\Gamma^{u} \cup \Gamma^{l}\right)} \Phi(\Lambda)\right\},
$$

which is called the weight of the partition function expansion (2.6). From [19], the last part in (2.6) can be expanded as follows:

$$
\exp \left\{-\sum_{\Lambda \subset \Lambda_{L, \infty}: \Lambda i\left(\Gamma^{u} \cup \Gamma^{l}\right)} \Phi(\Lambda)\right\}=\sum_{n=0}^{\infty} \sum_{\substack{\Lambda_{1}, \ldots, \Lambda_{n} \subset \Lambda_{L, \infty} \\ \Lambda_{v} i\left(\Gamma^{u} \cup \Gamma^{l}\right)}} \prod_{v=1}^{n}\left(e^{-\Phi\left(\Lambda_{v}\right)}-1\right) .
$$

In the definition of boundary condition $\omega^{b}$ (see (1.2)), for a fixed $b_{1} \geq 0$, let $\mathcal{S}_{L}=$ $\left(\cup_{b} \mathcal{S}_{L}^{b, u}, \cup_{b} \mathcal{S}_{L}^{b, l}\right)$, then, for the interfaces vector $\left(\Gamma^{u}, \Gamma^{l}\right) \in \mathcal{S}_{L}$, the height of the last ending 
point of $\left(\Gamma^{u}, \Gamma^{l}\right)$ is defined as the vector

$$
\left(h\left(\Gamma^{u}\right), h\left(\Gamma^{l}\right)\right)=\left(h\left(\pi\left(\Gamma^{u}\right)\right), h\left(\pi\left(\Gamma^{l}\right)\right)\right) .
$$

The aim of this paper is to study the statistical behaviors of the free energy of the height vector $\left(h\left(\Gamma^{u}\right), h\left(\Gamma^{l}\right)\right)$. The following theorem shows the limit existence of the free energy of the height for the interface vector $\left(\Gamma^{u}, \Gamma^{l}\right)$.

Theorem 2.1. For some $\delta>0$ and a complex vector $(\xi, \zeta) \in \mathbb{C}^{2}$, when the chemical potential $\mu$ is large enough, one has the existence of the free energy of the heights for the two-layered $W-R$ model as follows:

$$
\varphi(\xi, \zeta)=\lim _{L \rightarrow \infty} \frac{1}{L} \ln \sum_{\Gamma^{u}, \Gamma^{l} \in \mathcal{S}_{L}} e^{\mu\left(\xi h\left(\Gamma^{u}\right)+\zeta h\left(\Gamma^{l}\right)\right)} W\left(\Gamma^{u}, \Gamma^{l}\right)
$$

where the function $\varphi(\xi, \zeta)$ is analytic in $(\xi, \zeta) \in \mathbb{C}^{2}$ if the real parts $\operatorname{Re} \xi<1-\delta / \mu$ and $\operatorname{Re} \zeta<1-\delta / \mu$.

\section{Polymer Chains and Cluster Expansions}

Since the formula (2.11) heavily depends on the polymer representation of the two-layered $\mathrm{W}-\mathrm{R}$ model partition function and the theory of the cluster expansions, we study and analyze the polymer representation of the partition function in this section. And we show the estimate of the free energy for the height of the last ending point of $\left(\Gamma^{u}, \Gamma^{l}\right)$. Further, we show that the asymptotical behavior of the backbone $\pi\left(\Gamma^{u}\right)$ can represent that of the corresponding interface $\Gamma^{u}$ when $\mu$ is large enough; this means that the statistical properties of the interface $\Gamma^{u}$ are similar to those of its backbone $\pi\left(\Gamma^{u}\right)$. From the definition of $\pi\left(\Gamma^{u}\right)$, we define its polymer and polymer chains and develop a new polymer representation of the two-layered model partition function; we also obtain some estimates for the polymer weights.

For the Ising model, the polymers are defined by "cutting" the interface into elementary pieces at the line $\left\{x^{1}=n+1 / 2\right\}(n \in \mathbb{Z})$ of dual lattice, see [5-7]. However, this cutting procedure is invalid for the interface of the $\mathrm{W}$-R model, since the interface of the Ising model is a line, but the interface of the W-R model is a belt. So it needs to develop a new technique to cut the interface of the W-R model; that is, we hope to give a new definition of polymers. Higuchi et al. [2] introduce a new definition of polymers for one-layered interface of the W-R model; here we modify the definitions in [2] and define the polymer chains for the twolayered interface model.

For the interface vector $\left(\Gamma^{u}, \Gamma^{l}\right) \in \mathcal{S}_{L}$, let $\left(0, b_{1}\right)$ and $\left(L, k_{u}\right)$ be the starting and the ending points of $\Gamma^{u}$; let $\pi\left(\Gamma^{u}\right)$ be the backbone of $\Gamma^{u}$ connecting $\left(0, b_{1}\right)$ and $\left(L, k_{u}\right)$. Similarly, let $\left(0,-b_{1}\right)$ and $\left(L, k_{l}\right)$ be the starting and the ending points of $\Gamma^{l}$; let $\pi\left(\Gamma^{l}\right)$ be the backbone of $\Gamma^{l}$ connecting $\left(0,-b_{1}\right)$ and $\left(L, k_{l}\right)$. We decompose $\Gamma^{u} \backslash \pi\left(\Gamma^{u}\right)$ into connected components $\left\{C_{j}\right\}_{j=1}^{s}$ 
and decompose $\Gamma^{l} \backslash \pi\left(\Gamma^{l}\right)$ into connected components $\left\{D_{j}\right\}_{j=1}^{r}$. Then, from (2.6) and (2.9), we have

$$
\begin{aligned}
& \sum_{\Gamma^{u}, \Gamma^{l} \in \mathcal{S}_{L}} e^{\mu\left(\xi h\left(\Gamma^{u}\right)+\zeta h\left(\Gamma^{l}\right)\right)} W\left(\Gamma^{u}, \Gamma^{l}\right)
\end{aligned}
$$

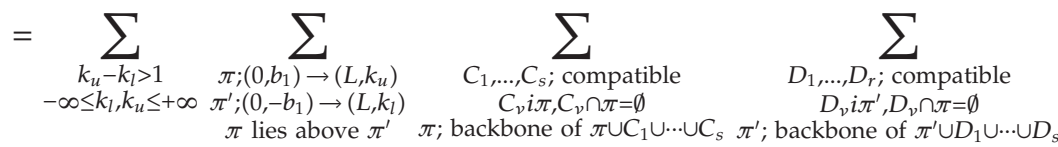

$$
\begin{aligned}
& \times \exp \left\{\mu \xi k_{u}-\mu|\pi|+N\left(\pi, C_{1}, \ldots, C_{s}\right) \ln 2-\mu \sum_{v=1}^{s}\left|C_{v}\right|\right\} \\
& \times \exp \left\{\mu \zeta k_{l}-\mu\left|\pi^{\prime}\right|+N\left(\pi^{\prime}, D_{1}, \ldots, D_{r}\right) \ln 2-\mu \sum_{v=1}^{r}\left|D_{v}\right|\right\} \sum_{\substack{\Lambda_{1}, \ldots, \Lambda_{t} ; \text { connected } \\
\Lambda_{\alpha} i \pi \cup C_{1} \cup \ldots \cup C_{s} \text { or } \\
\Lambda_{\alpha} i \pi^{\prime} \cup D_{1} \cup \cdots \cup D_{r}}} \prod_{\alpha=1}^{t}\left(e^{-\Phi\left(\Lambda_{\alpha}\right)}-1\right),
\end{aligned}
$$

where $N\left(\pi, C_{1}, \ldots, C_{s}\right)$ denotes the number of holes of $\pi \cup \cup_{v=1}^{s} C_{v}, N\left(\pi^{\prime}, D_{1}, \ldots, D_{r}\right)$ denotes the number of holes of $\pi^{\prime} \cup \cup_{v=1}^{r} D_{v}$, and the second summation taken over " $\pi$ lies above $\pi^{\prime \prime \prime}$, which is consistent with the definitions (i)-(iii) in Section 1.

Next we give the definitions of polymers of the two-layered W-R model. Let $a \leq c$ be the positive integers,

$$
\eta=\left(r^{u}, r^{l}, C_{1}, \ldots, C_{p}, D_{1}, \ldots, D_{q}, \Lambda_{1}, \ldots, \Lambda_{v}\right)
$$

is called a polymer with base $[a, c]$ if $\eta$ satisfies the following conditions (1)-(4).

(1) $\gamma^{u}, \gamma^{l}$ are self-avoiding paths in $\left\{a \leq x^{1} \leq c\right\}, \gamma^{u}$ starts from $\left(a, a^{u}\right)$ and ending at a point $\left(c, c^{u}\right)$ in $\left\{x^{1}=c\right\}, \gamma^{l}$ starts from $\left(a, a^{l}\right)$ and ending at a point $\left(c, c^{l}\right)$ in $\left\{x^{1}=c\right\}$, where $a^{u}, a^{l}, c^{u}, c^{l}$ are fixed integers satisfying $a^{u}-a^{l}>1$ and $c^{u}-c^{l}>1$, and $\gamma^{u}$ lies above $\gamma^{l}$.

(2) $\left\{C_{v}\right\}_{v=1}^{p}$ is a compatible family of connected subsets of $\left\{x \in \Lambda_{L, \infty} ; a \leq x^{1} \leq c\right\}$ such that (i) $C_{v} \cap V\left(\gamma^{u}\right)=\emptyset$, where $V\left(\gamma^{u}\right)$ is the set of vertices in $\gamma$; (ii) $C_{v} \cup V\left(\gamma^{u}\right)$ is connected; (iii) $\gamma^{u}$ is the backbone of $\gamma^{u} \cup C_{1} \cup \cdots \cup C_{p}$ with starting point $\left(a, a^{u}\right)$ and ending point $\left(c, c^{u}\right)$. Similarly, $\left\{D_{v}\right\}_{v=1}^{q}$ and $\gamma^{l}$ have the same properties.

(3) $\left\{\Lambda_{\alpha}\right\}_{\alpha=1}^{v}$ is a collection of connected subsets of $\left\{x \in \Lambda_{L, \infty} ; a \leq x^{1} \leq c\right\}$ such that

$$
\Lambda_{\alpha} i \gamma^{u} \cup \cup_{v=1}^{p} C_{v} \quad \text { or } \quad \Lambda_{\alpha} i \gamma^{l} \cup \cup_{v=1}^{q} D_{v}
$$

(4) For $a \leq j<c, j \in \mathbb{N}$, the line $\ell_{j}=\left\{x^{1}=j+1 / 2\right\}$ intersects at least two edges of $\gamma^{u} \cup \mathcal{E}\left(\cup_{v=1}^{p} C_{v} \cup \cup_{\alpha=1}^{v} \Lambda_{\alpha}\right) \cup \mathcal{E}\left(\gamma^{u}, \cup_{v=1}^{p} C_{v} \cup \cup_{\alpha=1}^{v} \Lambda_{\alpha}\right)$ or at least two edges of $\gamma^{l} \cup \mathcal{E}\left(\cup_{v=1}^{q} D_{v} \cup\right.$ $\left.\cup_{\alpha=1}^{v} \Lambda_{\alpha}\right) \cup \mathcal{E}\left(\gamma^{l}, \cup_{v=1}^{\bar{q}} D_{v} \cup \cup_{\alpha=1}^{v} \Lambda_{\alpha}\right)$. Here, for $B \subset \mathbb{Z}^{2}, \mathcal{E}(B)$ denotes the set of the nearest 
neighbor edges of $B ; \mathcal{\varepsilon}(\gamma, B)$ is the set of edges that connect $\gamma$ with the set $B$. Also, we identify an edge $\{x, y\}$ of $\mathbb{Z}^{2}$ with the line segment connecting $x$ and $y$.

We call $\left(\gamma^{u}, \gamma^{l}\right)$ the backbone of $\eta$. For two disjoint self-avoiding paths $\gamma_{1}^{u}, \gamma_{2}^{u}$ (similarly for $\gamma_{1}^{l}, \gamma_{2}^{l}$ ) such that the starting point of $\gamma_{2}^{u}$ is the nearest neighbor of the endpoint of $\gamma_{1}^{u}$, we can define the concatenation $\gamma_{1}^{u} \circ \gamma_{2}^{u}$ of these paths by simply connecting them. Let

$$
\begin{aligned}
& \eta_{1}=\left(r_{1}^{u}, r_{1}^{l}, C_{1}, \ldots, C_{p}, D_{1}, \ldots, D_{q}, \Lambda_{1}, \ldots, \Lambda_{v}\right), \\
& \eta_{2}=\left(r_{2}^{u}, r_{2}^{l}, C_{1}^{\prime}, \ldots, C_{u}^{\prime}, D_{1}^{\prime}, \ldots, D_{w}^{\prime}, \Lambda_{1}^{\prime}, \ldots, \Lambda_{z}^{\prime}\right)
\end{aligned}
$$

be two polymers with bases $[a, c]$ and $\left[a^{\prime}, c^{\prime}\right]\left(a \leq a^{\prime}\right)$, respectively. We say that $\eta_{1}$ and $\eta_{2}$ are compatible if either of the following conditions holds:

(a) $c+1<a^{\prime}$,

(b) $a^{\prime}=c+1$, the backbone of

$$
\begin{aligned}
\tilde{\Gamma}= & r_{1}^{u} \cup C_{1} \cup \cdots \cup C_{p} \cup\left(r_{2}^{u}+\left(0, h\left(r_{1}^{u}\right)\right)\right) \cup\left(C_{1}^{\prime}+\left(0, h\left(r_{1}^{u}\right)\right)\right) \cup \cdots \cup\left(C_{u}^{\prime}+\left(0, h\left(r_{1}^{u}\right)\right)\right) \\
& \cup r_{1}^{l} \cup D_{1} \cup \cdots \cup D_{q} \cup\left(r_{2}^{l}+\left(0, h\left(r_{1}^{l}\right)\right)\right) \cup\left(D_{1}^{\prime}+\left(0, h\left(r_{1}^{l}\right)\right)\right) \cup \cdots \cup\left(D_{w}^{\prime}+\left(0, h\left(r_{1}^{l}\right)\right)\right),
\end{aligned}
$$

is the concatenation $\left(\gamma_{1}^{u} \circ\left(\gamma_{2}^{u}+\left(0, h\left(\gamma_{1}^{u}\right)\right)\right), \gamma_{1}^{l} \circ\left(\gamma_{2}^{l}+\left(0, h\left(\gamma_{1}^{l}\right)\right)\right)\right)$, and connected components of the set $\tilde{\Gamma} \backslash\left(\gamma_{1}^{u} \circ\left(\gamma_{2}^{u}+\left(0, h\left(\gamma_{1}^{u}\right)\right)\right) \cup \gamma_{1}^{l} \circ\left(\gamma_{2}^{l}+\left(0, h\left(\gamma_{1}^{l}\right)\right)\right)\right)$ are $\left\{C_{1}, \ldots, C_{p}, C_{1}^{\prime}, \ldots, C_{u}^{\prime}\right\}$ and $\left\{D_{1}, \ldots, D_{q}, D_{1}^{\prime}, \ldots, D_{w}^{\prime}\right\}$. Here, $h(\gamma)$ is the height of the endpoint of $\gamma$.

Note that the previous work (see [2]) has presented the similar formula as that of the above (3.5) for the one-layered interface W-R model, so we can derive the formula (3.5) for the two-layered interface model from the corresponding work in Section 2 of [2].

The family $\left\{\eta_{p}\right\}_{p=0}^{n+1}$ is compatible if $\eta_{p}$ and $\eta_{p^{\prime}}\left(p \neq p^{\prime}\right)$ are compatible. Let

$$
\eta=\left(\pi\left(\Gamma^{u}\right), \pi\left(\Gamma^{l}\right), C_{1}, \ldots, C_{s}, D_{1}, \ldots, D_{r}, \Lambda_{1}, \ldots, \Lambda_{t}\right)
$$

An edge $e=\{x, y\}$ of $\eta$ is not admissible if it is a horizontal edge in $\mathcal{\varepsilon}\left(\pi\left(\Gamma^{u}\right), \cup_{v=1}^{s} C_{v} \cup\right.$ $\left.\cup_{\alpha=1}^{t} \Lambda_{\alpha}\right) \cup \mathcal{E}\left(\pi\left(\Gamma^{l}\right), \cup_{v=1}^{r} D_{v} \cup \cup_{\alpha=1}^{t} \Lambda_{\alpha}\right)$, such that

(i) the left vertex $x$ is in a connected $E$ of $\cup_{v=1}^{s} C_{v} \cup \cup_{v=1}^{r} D_{v} \cup \cup_{\alpha=1}^{t} \Lambda_{\alpha}$, and the right vertex $y$ is in $V\left(\pi\left(\Gamma^{u}\right)\right) \cup V\left(\pi\left(\Gamma^{u}\right)\right)$;

(ii) further, there exists a horizontal edge $e^{\prime}=\left\{x^{\prime}, y^{\prime}\right\}$ of $\eta$ such that $x^{\prime} \in V\left(\pi\left(\Gamma^{u}\right)\right) \cup$ $V\left(\pi\left(\Gamma^{u}\right)\right)$ and $y^{\prime} \in E$, where $x^{\prime}$ is the left vertex of $e^{\prime}$.

Other edges of $\eta$ are admissible.

We say that the line $\ell_{j}=\left\{x^{1}=j+1 / 2\right\}(0 \leq j \leq L-1)$ is the cutting line of $\eta$ if $\ell_{j}$ intersects only two admissible edges of $\eta=\left(\pi\left(\Gamma^{u}\right), \pi\left(\Gamma^{l}\right),\left\{C_{v}\right\}_{v=1}^{s},\left\{D_{v}\right\}_{v=1}^{r},\left\{\Lambda_{\alpha}\right\}_{\alpha=1}^{t}\right)$. Here, one of two admissible edges is connected to $\pi\left(\Gamma^{u}\right)$; the other is connected to $\pi\left(\Gamma^{l}\right)$. 
Let $\ell_{0}<\ell_{j_{1}}<\cdots<\ell_{j_{n}}<\ell_{j_{n+1}}=\ell_{L-1}$ be all the cutting lines of $\left(\pi\left(\Gamma^{u}\right), \pi\left(\Gamma^{l}\right),\left\{C_{v}\right\}_{v=1}^{s}\right.$, $\left.\left\{D_{v}\right\}_{v=1}^{r},\left\{\Lambda_{\alpha}\right\}_{\alpha=1}^{t}\right)$. For each $m \in\{0,1, \ldots, n+1\}$, there are only two edges $e_{m}^{u}=\left\{B_{m}^{u}, A_{m+1}^{u}\right\}$ and $e_{m}^{l}=\left\{B_{m}^{l}, A_{m+1}^{l}\right\}$ of $\pi\left(\Gamma^{u}\right)$, and $\pi\left(\Gamma^{l}\right)$, respectively, which intersect $\ell_{j_{m}}$. Let $\gamma_{m}^{u}$ be the portion of $\pi\left(\Gamma^{u}\right)$ starting from $A_{m}^{u}$ and ending at $B_{m}^{u}$; let $\gamma_{m}^{l}$ be the portion of $\pi\left(\Gamma^{l}\right)$ starting from $A_{m}^{l}$ and ending at $B_{m}^{l}$. Also let $\left\{C_{v}^{(m)}\right\}_{v=1}^{s(m)},\left\{D_{v}^{(m)}\right\}_{v=1}^{r(m)}$, and $\left\{\Lambda_{\alpha}^{(m)}\right\}_{\alpha=1}^{t(m)}$ be the set of elements of $\left\{C_{v}\right\}_{v=1}^{s}$, $\left\{D_{v}\right\}_{v=1}^{r}$, and $\left\{\Lambda_{\alpha}\right\}_{\alpha=1}^{t}$, respectively, such that they are subsets of $\left[j_{m-1}+1, j_{m}\right] \times(-\infty, \infty) \cap \mathbb{Z}^{2}$. Then $A_{m}^{u}=\left(j_{m-1}+1, p^{u}\right), A_{m}^{l}=\left(j_{m-1}+1, p^{l}\right)$ for some $p^{u}, p^{l} \in \mathbb{Z}$, where $p^{u}>p^{l}$. Thus, we obtain the $m$ th polymer $\eta_{m}$ by setting

$$
\eta_{m}=\left(r_{m}^{u}-\left(0, p^{u}\right), r_{m}^{l}-\left(0, p^{u}\right),\left\{C_{v}^{(m)}-\left(0, p^{u}\right)\right\}_{v=1}^{s(m)},\left\{D_{v}^{(m)}-\left(0, p^{u}\right)\right\}_{v=1}^{r(m)},\left\{\Lambda_{\alpha}^{(m)}-\left(0, p^{u}\right)\right\}_{\alpha=1}^{t(m)}\right) .
$$

By the above definitions, $\left\{\eta_{0}, \eta_{1}, \ldots, \eta_{n+1}\right\}$ are compatible.

For a polymer

$$
\eta_{m}=\left(\gamma_{m}^{u}, r_{m}^{l},\left\{C_{v}^{(m)}\right\},\left\{D_{v}^{(m)}\right\},\left\{\Lambda_{\alpha}^{(m)}\right\}\right)
$$

let $h_{m}^{u}=h\left(\eta_{m}^{u}\right)=h\left(\gamma_{m}^{u}\right)$ be the height of the endpoint of the self-avoiding path $\gamma_{m}^{u}$; similarly let $h_{m}^{l}=h\left(\eta_{m}^{l}\right)=h\left(\gamma_{m}^{l}\right)$. Then the heights $h\left(\pi\left(\Gamma^{u}\right)\right)$ and $h\left(\pi\left(\Gamma^{l}\right)\right)$ are given by

$$
h\left(\pi\left(\Gamma^{u}\right)\right)=\sum_{m=0}^{n+1} h\left(\gamma_{m}^{u}\right), \quad h\left(\pi\left(\Gamma^{l}\right)\right)=\sum_{m=0}^{n+1} h\left(\gamma_{m}^{l}\right) .
$$

Now we introduce a statistical weight of a polymer

$$
\eta^{\prime}=\left(\gamma^{u}, \gamma^{l},\left\{C_{v}\right\}_{v=1}^{p},\left\{D_{v}\right\}_{v=1}^{q},\left\{\Lambda_{\alpha}\right\}_{\alpha=1}^{v}\right)
$$

by setting

$$
\begin{gathered}
\Psi\left(\eta^{\prime}\right)=\exp \left\{-\mu\left|\gamma^{u}\right|-\mu\left|\gamma^{l}\right|+N^{*}\left(\gamma^{u}, C_{1}, \ldots, C_{p}\right) \ln 2+N^{*}\left(r^{l}, D_{1}, \ldots, D_{q}\right) \ln 2\right. \\
\left.-\mu \sum_{v=1}^{p}\left|C_{v}\right|-\mu \sum_{v=1}^{q}\left|D_{v}\right|\right\} \times \prod_{\alpha=1}^{v}\left(e^{-\Phi\left(\Lambda_{\alpha}\right)}-1\right),
\end{gathered}
$$

where

$$
N^{*}\left(\gamma^{u}, C_{1}, \ldots, C_{p}\right)=N\left(\gamma^{u}, C_{1}, \ldots, C_{p}\right)+N_{\hat{l}}\left(\gamma^{u}, C_{1}, \ldots, C_{p}\right)+N_{\widehat{r}}\left(\gamma^{u}, C_{1}, \ldots, C_{p}\right),
$$

and $N_{\hat{l}}\left(\gamma^{u}, C_{1}, \ldots, C_{p}\right)$ is the number of new holes created by $V\left(\gamma^{u}\right) \cup \cup_{v=1}^{p} C_{v}$ and the line $\left\{x^{1}=\right.$ $\widehat{l}-1\}$, where $\operatorname{base}\left(\eta^{\prime}\right)=[\widehat{l}, \widehat{r}] ; N_{\widehat{r}}\left(\gamma^{u}, C_{1}, \ldots, C_{p}\right)$ is the number of new holes created by $V\left(\gamma^{u}\right) \cup$ $\cup_{v=1}^{p} C_{v}$ and the line $\left\{x^{1}=\widehat{r}+1\right\}$. Similarly, we can give the definition of $N^{*}\left(\gamma^{l}, D_{1}, \ldots, D_{q}\right)$. 
A polymer $\eta$ is called simple if $\operatorname{base}(\eta)$ is one point and $\eta=\left(\gamma^{u}, \gamma^{l}, \emptyset, \emptyset, \emptyset\right)$. Thus, the weight $\Psi(\eta)$ is given by

$$
\Psi(\eta)=e^{-\mu\left|\gamma^{u}\right|-\mu\left|\gamma^{l}\right|}
$$

A polymer $\eta$ is called decorated if it is not simple. A decorated polymer $\eta=\left(\gamma^{u}, \gamma^{l},\left\{C_{v}\right\}\right.$, $\left.\left\{D_{v}\right\},\left\{\Lambda_{\alpha}\right\}\right)$ with $\operatorname{base}(\eta)=[\widehat{l}, \widehat{r}]$ is said to be $r$-active if there exists a simple polymer $\eta_{1}=\left(\gamma_{1}^{u}, r_{1}^{l}, \emptyset, \emptyset, \emptyset\right)$ with base $\left(\eta_{1}\right)=\{\hat{r}+1\}$ such that $\eta_{1}$ is incompatible with $\eta$, or the concatenation of $\gamma^{u}$ and $\gamma_{1}^{u}$ together with $\cup_{v} C_{v}$ produces a new hole, or the concatenation of $\gamma^{l}$ and $\gamma_{1}^{l}$ together with $\cup_{v} D_{v}$ produces a new hole. $\eta$ is said to be $l$-active if there exists a simple polymer $\eta_{2}=\left(\gamma_{2}^{u}, \gamma_{2}^{l}, \emptyset, \emptyset, \emptyset\right)$ with base $\left(\eta_{2}\right)=\{\widehat{r}-1\}$ such that $\eta_{2}$ is incompatible with $\eta_{\text {, the }}$ concatenation of $\gamma^{u}$ and $\gamma_{2}^{u}$ together with $\cup_{v} C_{v}$ produces a new hole, or the concatenation of $\gamma^{l}$ and $\gamma_{2}^{l}$ together with $\cup_{v} D_{v}$ produces a new hole. If $\eta$ is both $r$-active and $l$-active, we call it bi-active.

A polymer chain is a family of decorated polymers $\mathcal{C}=\left\{\eta_{1}, \ldots, \eta_{m}\right\}$ such that

(1) $\left\{\eta_{1}, \ldots, \eta_{m}\right\}$ are compatible;

(2) if $\operatorname{base}\left(\eta_{u}\right)=\left[\widehat{l}_{u}, \widehat{r}_{u}\right], 1 \leq u \leq m$, then $\widehat{l}_{u+1}=\widehat{r}_{u}+1$ or $\widehat{r}_{u}+2$ for each $u$;

(3) if $\widehat{l}_{u+1}=\widehat{r}_{u}+2$ for some $u$, then $\eta_{u}$ is $r$-active and $\eta_{u+1}$ is $l$-active.

Let $\mathcal{C}_{1}$ and $\mathcal{C}_{2}$ be two polymer chains. We say that $\mathcal{C}_{1}$ and $\mathcal{C}_{2}$ are compatible if $\mathcal{C}_{1} \cup \mathcal{C}_{2}$ is a compatible family of polymers, but now it is not a polymer chain. For example, if $\operatorname{base}\left(\mathcal{C}_{1}\right)=$ $\left[\widehat{l}_{u}, \widehat{r}_{u}\right]$ and base $\left(\mathcal{C}_{2}\right)=\left[\widehat{l}_{u}^{\prime}, \widehat{r}_{u}^{\prime}\right]$ have $\widehat{l}_{u}^{\prime}-\widehat{r}_{u}>2$, then $\mathcal{C}_{1} \cup \mathcal{C}_{2}$ is compatible polymers, but not a polymer chain.

Let $\mathcal{K}=\mathcal{K}_{L}$ be the set of all decorated polymers with base in $[0, L]$, and let $\mathcal{C} p_{L}$ denote the set of polymer chains with base in $[0, L]$, then we have the following Lemma 3.1.

Lemma 3.1. Let $Q(\xi, \zeta)$ be the generating function of the heights of the endpoints of a simple polymer

$$
Q(\xi, \zeta)=e^{-\mu} e^{-\mu} \sum_{\substack{k_{u}-k_{l}>1 \\-\infty \leq k_{l}, k_{u} \leq+\infty}} e^{\mu \xi k_{u}} e^{-\left|k_{u}\right| \mu} e^{\mu \zeta k_{l}} e^{-\left|k_{l}\right| \mu}
$$

then one has

$$
\frac{1}{Q(\xi, \zeta)^{L}} \sum_{\Gamma^{u}, \Gamma^{l} \in \mathcal{S}_{L}} e^{\mu\left(\xi h\left(\Gamma^{u}\right)+\zeta h\left(\Gamma^{l}\right)\right)} W\left(\Gamma^{u}, \Gamma^{l}\right)=\sum_{\begin{array}{c}
\mathcal{C}_{1}, \ldots, \mathcal{C}_{z} \in \mathcal{C} p_{L} ; \\
\text { compatible }
\end{array}} \prod_{i=1}^{z} \widehat{\Psi}\left(\mathcal{C}_{i} ; \xi, \zeta\right)
$$

where $\widehat{\Psi}\left(\mathcal{C}_{i}, \xi, \zeta\right)$ is the weight function of polymer chains which is given (3.17).

Proof of Lemma 3.1. Considering a polymer chain $\mathcal{C}=\left\{\eta_{1}, \ldots, \eta_{m}\right\}$, let base $(\mathcal{C})=\operatorname{base}\left(\eta_{1}\right) \cup$ $\cdots \cup$ base $\left(\eta_{m}\right)$. Further, for a polymer $\eta$, from (3.9) and (3.11), we define

$$
\widehat{\Psi}(\eta ; \xi, \zeta)=e^{\mu \xi h\left(\eta^{u}\right)} e^{\mu \zeta h\left(\eta^{l}\right)} \Psi(\eta) Q(\xi, \zeta)^{-|b a s e(\eta)|}
$$


where $|\operatorname{base}(\eta)|=\widehat{r}-\widehat{l}+1$ for $\operatorname{base}(\eta)=[\hat{l}, \widehat{r}]$. By the formula (3.16), for a polymer chain $\mathcal{C}=\left\{\eta_{1}, \ldots, \eta_{m}\right\}$, we put

$$
\widehat{\Psi}\left(\mathcal{C}_{i} ; \xi, \zeta\right)=\prod_{u=1}^{m} \widehat{\Psi}\left(\eta_{u} ; \xi, \zeta\right) \times \partial_{\hat{\imath}}\left(\eta_{1}\right) \partial_{\widehat{r}}\left(\eta_{m}\right) \prod_{u=1}^{m-1} \partial\left(\eta_{u}, \eta_{u+1}\right)
$$

where $\partial_{\hat{l}}, \partial_{\hat{r}}, \partial$ are defined in the following way. For base $(\eta)=[\hat{l}, \widehat{r}]$ and $\operatorname{base}(\bar{\eta})=[c, d]$ with $c>\widehat{r}$, we define

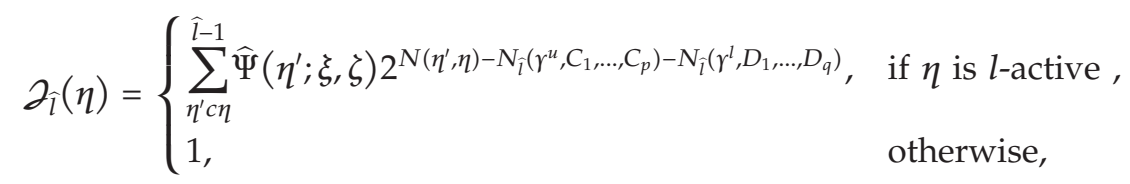

where $\sum_{\eta^{\prime} c \eta}^{\hat{l}-1}$ means over polymers $\eta^{\prime}=\left(\gamma^{\prime \prime}, \gamma^{\prime l}, \emptyset, \emptyset, \emptyset\right)$ with base $\{\hat{l}-1\}$ which are compatible with $\eta$, and $N\left(\eta^{\prime}, \eta\right)$ is the number of new holes created by the concatenation of $\gamma^{\prime u}$ and $\gamma^{u}$ together with $\cup_{v} C_{v}$ or by the concatenation of $\gamma^{\prime l}$ and $\gamma^{l}$ together with $\cup_{v} D_{v}$. Similarly,

$$
\partial_{\hat{r}}(\eta)= \begin{cases}\sum_{\eta^{\prime}}^{\hat{r}+1} \widehat{\Psi}\left(\eta^{\prime} ; \xi, \zeta\right) 2^{N\left(\eta, \eta^{\prime}\right)-N_{\hat{r}}\left(\gamma^{u}, C_{1}, \ldots, C_{p}\right)-N_{\hat{r}}\left(\gamma^{l}, D_{1}, \ldots, D_{q}\right),} & \text { if } \eta \text { is } r \text {-active }, \\ 1, & \text { otherwise, }\end{cases}
$$

and $2(\eta, \bar{\eta})$ is defined in the following two cases.

(1) If $c=\hat{r}+2, \eta$ is $r$-active and $\bar{\eta}$ is $l$-active, then

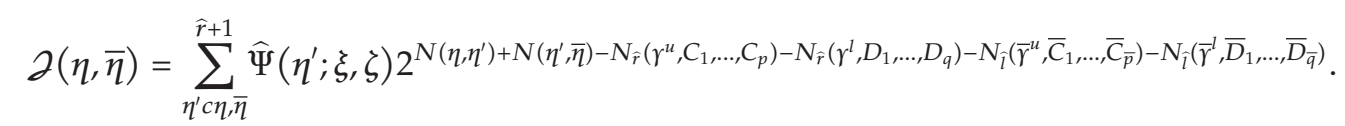

(2) If $c=\hat{r}+1, \eta$ and $\bar{\eta}$ are compatible, then

$$
2(\eta, \bar{\eta})=2^{N(\eta, \bar{\eta})-N_{\hat{r}}\left(\gamma^{u}, C_{1}, \ldots, C_{p}\right)-N_{\tilde{r}}\left(\gamma^{l}, D_{1}, \ldots, D_{q}\right)-N_{i}\left(\bar{\gamma}^{u}, \bar{C}_{1}, \ldots, \bar{C}_{\bar{p}}\right)-N_{\bar{l}}\left(\bar{\gamma}^{l}, \bar{D}_{1}, \ldots, \bar{D}_{\bar{q}}\right)}
$$

From the formulas (3.16) and (3.18)-(3.21), we show the weight expression of $\widehat{\Psi}\left(\mathcal{C}_{i} ; \xi, \zeta\right)$. According to the polymer representation of the partition function which is introduced in this section, and, by (3.1)-(3.14), we can show the existence of (3.15). This completes the proof of Lemma 3.1.

\section{Proof of the Main Results}

In the first part of this section, we do some preparations for the main results by some lemmas. Then we present the proof of Theorem 2.1. 
Lemma 4.1. Let $I \subset \mathbb{Z}$ be a fixed interval; if $\mu_{1}$ is large enough, then for some $\delta>0$, one has

$$
\sum_{\text {base }\left(\gamma^{u}\right)=I} e^{-(\delta / 3) N_{v}\left(\gamma^{u}\right)-\left(\mu_{1}-2 \delta / 3\right)\left(N_{h}\left(\gamma^{u}\right)+1\right)} \leq \frac{R\left(\mu_{1}, \delta\right)^{|I|}}{1-R\left(\mu_{1}, \delta\right)}
$$

where $\gamma^{u}$ is a upper backbone, $N_{v}\left(\gamma^{u}\right)$ is the number of vertical edges in $\gamma^{u}, N_{h}\left(\gamma^{u}\right)$ is the number of horizontal edges in $\gamma^{u}$, and

$$
R\left(\mu_{1}, \delta\right)=2 e^{-\left(\mu_{1}-2 \delta / 3\right)}\left(1+e^{-\delta / 3}\right)\left(1-e^{-\delta / 3}\right)^{-1}
$$

The upper bound of (4.1) also exists for the lower backbone $\gamma^{l}$.

Proof of Lemma 4.1. We separate $\gamma^{u}$ into fragments by the following method. Let $\gamma^{u}=\left\{x_{0}, x_{1}\right.$, $\left.\ldots, x_{n}\right\}$ be a self-avoiding path with $\operatorname{base}\left(\gamma^{u}\right)=I$. For $j_{0}=0$ and $i \geq 1$, we let

$$
j_{i}=\min \left\{j>j_{i-1} ;\left\{x_{j-1}, x_{j}\right\} \text { is a horizontal edge }\right\} .
$$

Each vertical part $\left\{x_{j_{i-1}}, x_{j_{i-1}+1}, \ldots, x_{j_{i}-1}\right\}$ of $\gamma^{u}$ with the direction of the exit vector $\left\{x_{j_{i}-1}, x_{j_{i}}\right\}$ is called a fragment. For a fragment $f=\left\{\bar{x}_{0}, \bar{x}_{1}, \ldots, \bar{x}_{p}\right\}$ with exit direction $e(f)$, we define

$$
W(f)=e^{-(\delta / 3) N_{v}(f)-\left(\mu_{1}-2 \delta / 3\right)}=e^{-p \delta / 3-\left(\mu_{1}-2 \delta / 3\right)} .
$$

Then the decomposition of $\gamma^{u}$ into fragments $\left\{f_{1}, \ldots, f_{r}\right\}$ leads to the identity

$$
e^{-(\delta / 3) N_{v}\left(\gamma^{u}\right)-\left(\mu_{1}-2 \delta / 3\right)\left(N_{h}\left(\gamma^{u}\right)+1\right)}=\prod_{j=1}^{r} W\left(f_{j}\right) .
$$

Therefore, if $\mu_{1}$ is sufficiently large, we have

$$
\begin{aligned}
\sum_{r^{u} ; \text { base }\left(\gamma^{u}\right)=I} e^{-(\delta / 3) N_{v}\left(\gamma^{u}\right)-\left(\mu_{1}-2 \delta / 3\right)\left(N_{h}\left(\gamma^{u}\right)+1\right)} & =\sum_{r=|I|}^{\infty} \sum_{f_{1}, \ldots, f_{r}} \prod_{j=1}^{r} W\left(f_{j}\right) \\
& \leq \sum_{r=|I|}^{\infty}\left(2 \sum_{k=-\infty}^{\infty} e^{-(\delta / 3)|k|}\right)^{r} \times e^{-\left(\mu_{1}-2 \delta / 3\right) r} \\
& =\frac{R\left(\mu_{1}, \delta\right)^{|I|}}{1-R\left(\mu_{1}, \delta\right)}
\end{aligned}
$$

where the last equality comes from [7]. Following the same proving procedure, we can obtain the existence of the formula (4.1) for $\gamma^{l}$. This completes the proof of Lemma 4.1. 
Lemma 4.2. There is a large chemical potential $\mu_{2}>0$; if $\mu>\mu_{2}$, then for any $\xi, \zeta \in \mathbb{C}$ with the conditions $\operatorname{Re} \xi<1-\delta / \mu$ and $\operatorname{Re} \zeta<1-\delta / \mu$, and for each polymer $\eta_{0}$, one has

$$
\sum_{\eta \in \mathcal{K}_{L} ; \eta i \eta_{0}} e^{c(\eta)+d(\eta)}|\widehat{\Psi}(\eta ; \xi, \zeta)| \leq c\left(\eta_{0}\right)
$$

where $\mathcal{K}_{L}$ is the set of all decorated polymers with the base in $[0, L], c(\eta)=3 \mid$ base $(\eta) \mid$, and

$$
d(\eta)= \begin{cases}\left(\mu-\mu_{2}\right)|\operatorname{base}(\eta)|+\frac{\delta}{6}\left|\gamma^{u}\right|+\frac{\delta}{6}\left|\gamma^{l}\right|-\left(\mu-\mu_{1}-1\right), & \text { if }|\operatorname{base}(\eta)| \geq 2 \\ \left(\mu-\mu_{2}\right)|\operatorname{base}(\eta)|+\frac{\delta}{6}\left|\gamma^{u}\right|+\frac{\delta}{6}\left|\gamma^{l}\right|, & \text { if }|\operatorname{base}(\eta)|=1\end{cases}
$$

where $\mu_{1}$ is given in Lemma 4.1 .

Proof of Lemma 4.2. In order to verify the convergence and analyticity, we have to show that there exist two functions

$$
c, d: \mathcal{K}_{L}=\{\xi ; \text { decorated polymer }\} \longrightarrow[0, \infty),
$$

such that the above estimate (4.7) exists. First we consider the statistical properties of

$$
\eta=\left(\gamma^{u}, \gamma^{l}, C_{1}, \ldots, C_{p}, D_{1}, \ldots, D_{q}, \Lambda_{1}, \ldots, \Lambda_{v}\right)
$$

and we have

$$
\left|r^{u}\right|=N_{v}\left(r^{u}\right)+N_{h}\left(r^{u}\right)+1, \quad\left|r^{l}\right|=N_{v}\left(r^{l}\right)+N_{h}\left(r^{l}\right)+1
$$

By the definition of the decorated polymers, if $\operatorname{base}(\eta)$ is one point, then

$$
N_{h}\left(\gamma^{u}\right)+N_{h}\left(\gamma^{l}\right)+\sum_{v=1}^{p}\left|C_{v}\right|+\sum_{v=1}^{q}\left|D_{v}\right|+\sum_{\alpha=1}^{v}\left|\Lambda_{\alpha}\right| \geq 1
$$

since $\left\{C_{v}\right\}$ or $\left\{D_{v}\right\}$ or $\left\{\Lambda_{\alpha}\right\}$ is nonempty if $\operatorname{base}(\eta)$ is one point. If $|\operatorname{base}(\eta)| \geq 2$, then we have

$$
N_{h}\left(\gamma^{u}\right)+N_{h}\left(\gamma^{l}\right)+\sum_{v=1}^{p}\left|C_{v}\right|+\sum_{v=1}^{q}\left|D_{v}\right|+\sum_{\alpha=1}^{v}\left|\Lambda_{\alpha}\right| \geq 3(\mid \text { base }(\xi) \mid-1) .
$$

Let $\left(\gamma^{u}, \gamma^{l}\right)$ be the backbone of some decorated polymer with the base $I=[\hat{l}, \widehat{r}]$. Next we estimate the following function

$$
G\left(\gamma^{u}, \gamma^{l}\right)=\sum_{\eta ;\left(\gamma^{u}, \gamma^{l}\right)} \text { is the backbone of } \eta
$$


From (2.4) and $|\Phi(\Lambda)| \leq e^{-\left(\mu-\mu_{0}\right)|\Lambda|}<1$ for large parameter $\mu_{0}$, we have

$$
\left|e^{-\Phi(\Lambda)}-1\right| \leq e^{\left(-\mu-\mu_{0}-1\right)|\Lambda|}
$$

(i) If $\widehat{l}=\widehat{r}$, that is $|I|=1$, then

$$
N^{*}\left(\gamma^{u}, C_{1}, \ldots, C_{p}\right)=N^{*}\left(r^{l}, D_{1}, \ldots, D_{q}\right)=0 .
$$

From the (3.11), (4.14), and (4.15), we have

$$
\begin{aligned}
& G\left(\gamma^{u}, \gamma^{l}\right) \leq e^{-\mu\left|\gamma^{u}\right|} e^{\mu h\left(\gamma^{u}\right) \operatorname{Re} \xi} \sum_{\left\{C_{v}\right\} ; C_{\nu} i \gamma^{u}} e^{-\mu \sum_{\nu}\left|C_{v}\right|} \times e^{-\mu\left|\gamma^{l}\right|} e^{\mu h\left(\gamma^{l}\right) \operatorname{Re} \zeta} \sum_{\left\{D_{v}\right\} ; D_{v} i \gamma^{l}} e^{-\mu \sum_{v}\left|D_{v}\right|} \\
& \times \sum_{\substack{\left\{\Lambda_{\alpha}\right\} ; \Lambda_{\alpha} i \gamma^{u} \cup C_{1} \cup \ldots \cup C_{p} \\
\text { or } \Lambda_{\alpha} i \gamma^{\cup} \cup D_{1} \cup \cdots \cup D_{q}}} e^{-\left(\mu-\mu_{0}-1\right) \sum_{\alpha}\left|\Lambda_{\alpha}\right|} \\
& \leq e^{-\mu\left|\gamma^{u}\right|+\mu h\left(\gamma^{u}\right) \operatorname{Re} \xi-\mu\left|\gamma^{l}\right|+\mu h\left(\gamma^{l}\right) \operatorname{Re} \zeta-\left(\mu-\mu_{1}-1\right)} \sum_{\left\{C_{v}\right\} ; C_{v} i \gamma^{u}} e^{-\mu_{1} \sum_{v}\left|C_{v}\right|} \\
& \times \sum_{\left\{D_{\nu}\right\} ; D_{\nu} i \gamma^{l}} e^{-\mu_{1} \Sigma_{\nu}\left|D_{v}\right|} \sum_{\substack{\left\{\Lambda_{\alpha}\right\} ; \Lambda_{\alpha} i \gamma^{u} \cup C_{1} \cup \cdots \cup C_{p} \\
\text { or } \Lambda_{\alpha} i \gamma^{l} \cup D_{1} \cup \cdots \cup D_{q}}} e^{-\left(\mu_{1}-\mu_{0}\right) \sum_{\alpha}\left|\Lambda_{\alpha}\right|} .
\end{aligned}
$$

The summation over $\left\{\Lambda_{\alpha}\right\}$ is estimated as follows:

$$
\begin{aligned}
& \sum_{\left\{\Lambda_{\alpha}\right\} ; \Lambda_{\alpha} i \gamma^{u} \cup C_{1} \cup \ldots \cup C_{p}} e^{-\left(\mu_{1}-\mu_{0}\right) \sum_{\alpha}\left|\Lambda_{\alpha}\right|} \\
& \text { or } \Lambda_{\alpha} i \gamma^{l} \cup D_{1} \cup \cdots \cup D_{q} \\
& \leq \sum_{t=0}^{\infty} \frac{1}{t !} \sum_{\substack{\Lambda_{1} i \gamma^{u} \cup C_{1} \cup \cdots \cup C_{p} \\
\text { or } \Lambda_{1} i \gamma^{l} \cup D_{1} \cup \cdots \cup D_{q}}} \cdots \sum_{\begin{array}{c}
\Lambda_{t} i \gamma^{u} \cup C_{1} \cup \cdots \cup C_{p} \\
\text { or } \Lambda_{t} i \gamma^{l} \cup D_{1} \cup \cdots \cup D_{q}
\end{array}} e^{-\left(\mu_{1}-\mu_{0}\right) \sum_{\alpha}\left|\Lambda_{\alpha}\right|} \\
& \leq \exp \left\{4\left|\gamma^{u} \cup C_{1} \cup \cdots \cup C_{p} \cup \gamma^{l} \cup D_{1} \cup \cdots \cup D_{q}\right| \sum_{\Lambda \ni 0 ; \text { connected }} e^{-\left(\mu_{1}-\mu_{0}\right)|\Lambda|}\right\} \\
& =\exp \left\{\left(\left|\gamma^{u}\right|+\left|\gamma^{l}\right|+\sum_{v}\left|C_{v}\right|+\sum_{v}\left|D_{v}\right|\right) g_{1}\left(\mu_{1}, \mu_{0}\right)\right\} .
\end{aligned}
$$

There exist constants $K_{1}, \kappa>0$ such that the number $N_{m}$ of connected sets of $m$ points in $\mathbb{Z}^{2}$ which contain the origin is bounded as

$$
N_{m} \leq K_{1} \kappa^{m} \quad(m \geq 1),
$$


Abstract and Applied Analysis

then we know that the function

$$
g_{1}\left(\mu_{1}, \mu_{0}\right)=4 \sum_{\Lambda \ni 0 ; \text { connected }} e^{-\left(\mu_{1}-\mu_{0}\right)|\Lambda|},
$$

goes to zero exponentially fast as $\mu_{1} \rightarrow \infty$. Thus, from (4.17) and (4.18) we obtain

$$
\begin{aligned}
G\left(\gamma^{u}, \gamma^{l}\right) \leq & e^{-\left(\mu-g_{1}\left(\mu_{1}, \mu_{0}\right)\right)\left|\gamma^{u}\right|+\mu h\left(\gamma^{u}\right) \operatorname{Re} \xi^{-}-\left(\mu-g_{1}\left(\mu_{1}, \mu_{0}\right)\right)\left|\gamma^{l}\right|+\mu h\left(\gamma^{l}\right) \operatorname{Re} \zeta} \times e^{-\left(\mu-\mu_{1}-1\right)} \\
& \times \sum_{\left\{C_{v}\right\} ; C_{v} i \gamma^{u}} e^{-\left(\mu_{1}-g_{1}\left(\mu_{1}, \mu_{0}\right)\right) \sum_{v}\left|C_{v}\right|} \sum_{\left\{D_{v}\right\} ; D_{v} i \gamma^{l}} e^{-\left(\mu_{1}-g_{1}\left(\mu_{1}, \mu_{0}\right)\right) \sum_{v}\left|D_{v}\right|} \\
\leq & e^{-\left(\mu-g_{1}\left(\mu_{1}, \mu_{0}\right)-g_{2}\left(\mu_{1}, \mu_{0}\right)\right)\left|\gamma^{u}\right|+\mu h\left(\gamma^{u}\right) \operatorname{Re} \xi} \\
& \times e^{-\left(\mu-g_{1}\left(\mu_{1}, \mu_{0}\right)-g_{2}\left(\mu_{1}, \mu_{0}\right)\right)\left|\gamma^{l}\right|+\mu h\left(\gamma^{l}\right) \operatorname{Re} \zeta} \times e^{-\left(\mu-\mu_{1}-1\right)}
\end{aligned}
$$

where

$$
g_{2}\left(\mu_{1}, \mu_{0}\right)=4 \sum_{D \ni 0 ; \text { connected }} e^{-\left(\mu_{1}-g_{1}\left(\mu_{1}, \mu_{0}\right)\right)|D|}
$$

(ii) If $\widehat{l}<\widehat{r}$; that is, $|I| \geq 2$, then we have

$$
\begin{aligned}
& N^{*}\left(\gamma^{u}, C_{1}, \ldots, C_{p}\right) \leq N_{h}\left(\gamma^{u}\right)+\sum_{v=1}^{p}\left|C_{v}\right|, \\
& N^{*}\left(\gamma^{l}, D_{1}, \ldots, D_{q}\right) \leq N_{h}\left(\gamma^{l}\right)+\sum_{v=1}^{q}\left|D_{v}\right| .
\end{aligned}
$$

From the formula (4.13), we have

$$
\begin{aligned}
& G\left(\gamma^{u}, \gamma^{l}\right) \leq e^{-\mu\left|\gamma^{u}\right|} e^{\mu h\left(\gamma^{u}\right) \operatorname{Re} \xi} e^{-\mu\left|\gamma^{l}\right|} e^{\mu h\left(\gamma^{l}\right) \operatorname{Re} \zeta} \sum_{\left\{C_{v}\right\} ; C_{\nu} i \gamma^{u}} e^{-\mu \sum_{\nu}\left|C_{\nu}\right| 2^{N^{*}}\left(\gamma^{u}, C_{1}, \ldots, C_{p}\right)} \\
& \times \sum_{\left\{D_{v}\right\} ; D_{v} i \gamma^{l}} e^{-\mu \sum_{v}\left|D_{\nu}\right| 2^{N^{*}}\left(\gamma^{l}, D_{1}, \ldots, D_{q}\right)} \sum_{\substack{\left\{\Lambda_{\alpha}\right\} ; \Lambda_{\alpha} i \gamma^{u} \cup C_{1} \cup \cdots \cup C_{p} \\
\text { or } \Lambda_{\alpha} i \gamma^{l} \cup D_{1} \cup \cdots \cup D_{q}}} e^{-\left(\mu-\mu_{0}-1\right) \sum_{\alpha}\left|\Lambda_{\alpha}\right|} \\
& \leq e^{-\mu\left|\gamma^{u}\right|+\mu h\left(\gamma^{u}\right) \operatorname{Re} \xi-\mu\left|\gamma^{l}\right|+\mu h\left(\gamma^{l}\right) \operatorname{Re} \zeta-\left(\mu-\mu_{1}-1\right)\left(3|I|-N_{h}\left(\gamma^{u}\right)-N_{h}\left(\gamma^{l}\right)-3\right)} 2^{N_{h}\left(\gamma^{u}\right)} 2^{N_{h}\left(\gamma^{l}\right)} \\
& \times \sum_{\left\{C_{\nu}\right\} ; C_{\nu} i \gamma^{u}} e^{-\left(\mu_{1}-\ln 2\right) \sum_{\nu}\left|C_{\nu}\right|} \sum_{\left\{D_{\nu}\right\} ; D_{\nu} i \gamma^{l}} e^{-\left(\mu_{1}-\ln 2\right) \sum_{v}\left|D_{v}\right|} \\
& \times \sum_{\substack{\left\{\Lambda_{\alpha}\right\} ; \Lambda_{\alpha} i \gamma^{u} \cup C_{1} \cup \cdots \cup C_{p} \\
\text { or } \Lambda_{\alpha} i \gamma \gamma^{\cup} \cup D_{1} \cup \cdots \cup D_{q}}} e^{-\left(\mu_{1}-\mu_{0}\right) \sum_{\alpha}\left|\Lambda_{\alpha}\right|} .
\end{aligned}
$$


Further, according to the formula (4.18), we have

$$
\begin{aligned}
& G\left(\gamma^{u}, \gamma^{l}\right) \leq e^{-\left(\mu-g_{1}\left(\mu_{1}, \mu_{0}\right)\right)\left|\gamma^{u}\right|+\mu h\left(\gamma^{u}\right) \operatorname{Re} \xi-\left(\mu-g_{1}\left(\mu_{1}, \mu_{0}\right)\right)\left|\gamma^{l}\right|+\mu h\left(\gamma^{l}\right) \operatorname{Re} \zeta} e^{-\left(\mu-\mu_{1}-1\right)\left(3|I|-N_{h}\left(\gamma^{u}\right)-N_{h}\left(\gamma^{l}\right)-3\right)} \\
& \times \sum_{\left\{C_{v}\right\} ; C_{\nu} i \gamma^{u}} e^{-\left(\mu_{1}-g_{1}\left(\mu_{1}, \mu_{0}\right)-\ln 2\right) \sum_{v}\left|C_{\nu}\right|} 2^{N_{h}\left(\gamma^{u}\right)} \sum_{\left\{D_{v}\right\} ; D_{v} i \gamma^{l}} e^{-\left(\mu_{1}-g_{1}\left(\mu_{1}, \mu_{0}\right)-\ln 2\right) \sum_{v}\left|D_{v}\right| 2^{N_{h}\left(\gamma^{l}\right)}} \\
& \leq e^{-\left(\mu-g_{1}\left(\mu_{1}, \mu_{0}\right)-g_{3}\left(\mu_{1}, \mu_{0}\right)\right)\left|\gamma^{u}\right|+\mu h\left(\gamma^{u}\right) \operatorname{Re} \xi} \times e^{-\left(\mu-g_{1}\left(\mu_{1}, \mu_{0}\right)-g_{3}\left(\mu_{1}, \mu_{0}\right)\right)\left|\gamma^{l}\right|+\mu h\left(\gamma^{l}\right) \operatorname{Re} \zeta} \\
& \times e^{-\left(\mu-\mu_{1}-1\right)\left(3|I|-N_{h}\left(\gamma^{u}\right)-N_{h}\left(\gamma^{l}\right)-3\right)+N_{h}\left(\gamma^{u}\right) \ln 2+N_{h}\left(\gamma^{l}\right) \ln 2,}
\end{aligned}
$$

where

$$
g_{3}\left(\mu_{1}, \mu_{0}\right)=4 \sum_{D \ni 0 ; \text { connected }} e^{-\left(\mu_{1}-g_{1}\left(\mu_{1}, \mu_{0}\right)-\ln 2\right)|D|} .
$$

We choose $\mu_{1}\left(>\mu_{0}\right)$ sufficiently large, such that

$$
g_{1}\left(\mu_{1}, \mu_{0}\right)<\frac{\delta}{4}, \quad g_{2}\left(\mu_{1}, \mu_{0}\right)<\frac{\delta}{4}, \quad g_{3}\left(\mu_{1}, \mu_{0}\right)<\frac{\delta}{4} .
$$

Assume that $\operatorname{Re} \xi<1-\delta / \mu$ and $\operatorname{Re} \zeta<1-\delta / \mu$. Since

$$
N_{v}\left(\gamma^{u}\right) \geq\left|h\left(\gamma^{u}\right)\right|, \quad N_{v}\left(r^{l}\right) \geq\left|h\left(r^{l}\right)\right|,
$$

then from the formula (4.11), for $|I| \geq 2$, we have

$$
G\left(\gamma^{u}, \gamma^{l}\right) \leq e^{-(\delta / 2) N_{v}\left(\gamma^{u}\right)-\left(\mu_{1}-\delta / 2\right)\left(N_{h}\left(\gamma^{u}\right)+1\right)} e^{-(\delta / 2) N_{v}\left(\gamma^{l}\right)-\left(\mu_{1}-\delta / 2\right)\left(N_{h}\left(\gamma^{l}\right)+1\right)} e^{-\left(\mu-\mu_{1}-1\right)(3|I|-1)}
$$

For $|I|=1$, we have

$$
G\left(\gamma^{u}, \gamma^{l}\right) \leq e^{-(\delta / 2) N_{v}\left(\gamma^{u}\right)-(\delta / 2) N_{v}\left(\gamma^{u}\right)-2\left(\mu_{1}-\delta / 2\right)} e^{-3\left(\mu-\mu_{1}-1\right)} .
$$

Let $c(\eta), d(\eta)$ be defined in (4.7). Since $c(\eta)$ and $d(\eta)$ only depend on the backbone $\left(\gamma^{u}, \gamma^{l}\right)$, let

$$
c(\eta)=c\left(r^{u}, \gamma^{l}\right), \quad d(\eta)=d\left(\gamma^{u}, \gamma^{l}\right) .
$$


Then, for a fixed interval $I$, we have

$$
\begin{aligned}
& \quad \sum_{\eta ;\left(\gamma^{u}, \gamma^{l}\right) \text { is the backbone of } \eta} e^{c(\eta)+d(\eta)}\left|e^{\mu \xi h\left(\gamma^{u}\right)} e^{\mu \xi h\left(\gamma^{l}\right)} \Psi(\eta)\right| \\
& =G\left(\gamma^{u}, \gamma^{l}\right) e^{c\left(\gamma^{u}, \gamma^{l}\right)+d\left(\gamma^{u}, \gamma^{l}\right)} \\
& \leq e^{-(\delta / 3) N_{v}\left(\gamma^{u}\right)-\left(\mu_{1}-2 \delta / 3\right)\left(N_{h}\left(\gamma^{u}\right)+1\right)} e^{-(\delta / 3) N_{v}\left(\gamma^{l}\right)-\left(\mu_{1}-2 \delta / 3\right)\left(N_{h}\left(\gamma^{l}\right)+1\right)} e^{-\left(2 \mu+\mu_{2}-3 \mu_{1}-6\right)\left|b a s e\left(\gamma^{u}, \gamma^{l}\right)\right|},
\end{aligned}
$$

where $\operatorname{base}\left(\gamma^{u}, \gamma^{l}\right)=\operatorname{base}(\eta)$ for any $\eta$ such that $\left(\gamma^{u}, \gamma^{l}\right)$ is the backbone of $\eta$. Then

$$
\begin{aligned}
& \sum_{\operatorname{base}(\gamma)=I} e^{c\left(\gamma^{u}, \gamma^{l}\right)+d\left(\gamma^{u}, \gamma^{l}\right)} G\left(\gamma^{u}, \gamma^{l}\right) \\
& \leq e^{-\left(2 \mu+\mu_{2}-3 \mu_{1}-6\right)|I|} \sum_{\text {base }(\gamma)=I} e^{-(\delta / 3) N_{v}\left(\gamma^{u}\right)-\left(\mu_{1}-2 \delta / 3\right)\left(N_{h}\left(\gamma^{u}\right)+1\right)} e^{-(\delta / 3) N_{v}\left(\gamma^{l}\right)-\left(\mu_{1}-2 \delta / 3\right)\left(N_{h}\left(\gamma^{l}\right)+1\right)} .
\end{aligned}
$$

By Lemma 4.1, if $\operatorname{Re} \xi<1-\delta / \mu, \operatorname{Re} \zeta<1-\delta / \mu$, and $\mu>\mu_{1}$, where $\mu_{1}$ is sufficiently large, we have

$$
\sum_{\operatorname{base}(\xi)=I} e^{c\left(\gamma^{u}, \gamma^{l}\right)+d\left(\gamma^{u}, \gamma^{l}\right)} G\left(\gamma^{u}, \gamma^{l}\right) \leq e^{-\left(2 \mu+\mu_{2}-3 \mu_{1}-6\right)|I|}\left(\frac{R\left(\mu_{1}, \delta\right)^{|I|}}{1-R\left(\mu_{1}, \delta\right)}\right)^{2}
$$

From (3.14), there is a $\mu_{3}>0$ such that

$$
|Q(\xi, \zeta)| \geq e^{-2 \mu-\mu_{3}}
$$

then we have

$$
\begin{aligned}
\sum_{\operatorname{base}(\xi)=I}|\widehat{\Psi}(\eta ; \xi, \zeta)| e^{c\left(\gamma^{u}, \gamma^{l}\right)+d\left(\gamma^{u}, \gamma^{l}\right)} & \leq e^{-\left(2 \mu+\mu_{2}-3 \mu_{1}-6\right)|I|}\left(\frac{R\left(\mu_{1}, \delta\right)^{|I|}}{1-R\left(\mu_{1}, \delta\right)}\right)^{2} e^{\left(2 \mu+\mu_{3}\right)|I|} \\
& =e^{-\left(\mu_{2}-3 \mu_{1}-\mu_{3}-6\right)|I|}\left(\frac{R\left(\mu_{1}, \delta\right)^{|I|}}{1-R\left(\mu_{1}, \delta\right)}\right)^{2} .
\end{aligned}
$$


Let $\mu_{1}$ be large enough, we take $\mu_{2}>3 \mu_{1}+\mu_{3}+6$ and $\mu>\mu_{2}$. For a fixed $\eta_{0}$ with the $\operatorname{base}\left(\eta_{0}\right)=$ $[\widehat{l}, \widehat{r}]$, then we have

$$
\begin{aligned}
\sum_{\eta i \eta_{0}}|\widehat{\Psi}(\eta ; \xi, \zeta)| e^{c(\eta)+d(\eta)} & \leq \sum_{x \in[\hat{l}-1, \hat{r}+1]} \sum_{I \ni x}\left(\frac{R\left(\mu_{1}, \delta\right)^{|I|}}{1-R\left(\mu_{1}, \delta\right)}\right)^{2} \\
& =\frac{(\widehat{r}-\widehat{l}+3)}{\left(1-R\left(\mu_{1}, \delta\right)\right)^{2}} \sum_{k=1}^{\infty} k R\left(\mu_{1}, \delta\right)^{2 k} \\
& \leq \frac{3\left|b a s e\left(\eta_{0}\right)\right|}{\left(1-R\left(\mu_{1}, \delta\right)\right)^{2}} \times \frac{R\left(\mu_{1}, \delta\right)^{2}}{\left(1-R\left(\mu_{1}, \delta\right)^{2}\right)^{2}} \\
& \leq c\left(\eta_{0}\right) .
\end{aligned}
$$

This finishes the proof of Lemma 4.2.

Next we give the proof of Theorem 2.1, where the technique of polymer chains and Lemmas 3.1-4.2 are applied to show the limit existence of the free energy for the two-layered lattice W-R model.

Proof of Theorem 2.1. From Lemma 3.1, we have

$$
\frac{1}{Q(\xi, \zeta)^{L}} \sum_{\Gamma^{u}, \Gamma^{l} \in \mathcal{S}_{L}} e^{\mu\left(\xi h\left(\Gamma^{u}\right)+\zeta h\left(\Gamma^{l}\right)\right)} W\left(\Gamma^{u}, \Gamma^{l}\right)=\sum_{\begin{array}{c}
\mathcal{C}_{1}, \ldots, \mathcal{C}_{z} \in \mathcal{C} p_{L} ; \\
\text { compatible }
\end{array}} \prod_{i=1}^{z} \widehat{\Psi}\left(\mathcal{C}_{i} ; \xi, \zeta\right) .
$$

In the following part, we will show the existence of the following limit:

$$
\widehat{\varphi}(\xi, \zeta)=\lim _{L \rightarrow \infty} \frac{1}{L} \ln \sum_{\substack{\mathcal{C}_{1}, \ldots, \mathcal{C}_{z} \in \mathcal{C} p_{L} ; \\ \text { compatible }}} \prod_{i=1}^{z} \widehat{\Psi}\left(\mathcal{C}_{i} ; \xi, \zeta\right)
$$

where $\widehat{\varphi}(\xi, \zeta)$ is analytic for $\operatorname{Re} \xi<1-\delta / \mu$ and $\operatorname{Re} \zeta<1-\delta / \mu$. In this case, from the formulas (2.11), (4.38), and (4.39) we have

$$
\varphi(\xi, \zeta)=\widehat{\varphi}(\xi, \zeta)+\ln Q(\xi, \zeta)
$$

which is analytic in this region. According to (4.40), in order to demonstrate Theorem 2.1 (or to prove the existence of (2.11)), it is sufficient to show the limit existence of the above (4.39).

Next we estimate the weight of the polymer chains. We call a family of intervals

$$
I_{1}=\left[\widehat{l}_{1}, \widehat{r}_{1}\right], \ldots, I_{n}=\left[\widehat{l}_{n}, \widehat{r}_{n}\right]
$$


the linked intervals if $\widehat{r}_{u}<\widehat{l}_{u+1}<\widehat{r}_{u}+2$ for each $1 \leq u \leq n$. So that the base of a polymer chain forms the linked intervals. For a fixed polymer chain $\mathcal{C}_{0}$, let $\left[\right.$ base $\left.\left(\mathcal{C}_{0}\right)\right]=\left[\widehat{l}_{0}, \widehat{r}_{0}\right]$ be the smallest intervals (in length) including base $\left(\mathcal{C}_{0}\right)$. Let

$$
c^{*}(\mathcal{C})=\sum_{\eta \in \mathcal{C}} c(\eta), \quad d^{*}(\mathcal{C})=\sum_{\eta \in \mathcal{C}} d(\eta)
$$

where $c(\eta)$ and $d(\eta)$ are defined in Lemma 4.2. Then, for the functions

$$
c^{*}, d^{*}: \mathcal{C} D=\{\mathcal{C} \text {; polymer chain }\} \longrightarrow[0, \infty),
$$

we show that

$$
\sum_{\mathcal{C} \in \mathcal{C} p_{L} ; \mathcal{C} i \mathcal{C}_{0}}|\widehat{\Psi}(\mathcal{C} ; \xi, \zeta)| e^{c^{*}(\mathcal{C})+d^{*}(\mathcal{C})} \leq c^{*}\left(\mathcal{C}_{0}\right)
$$

for any polymer chain $\mathcal{C}_{0}$ and for any $\xi, \zeta \in \mathbb{C}$ with $\operatorname{Re} \xi<1-\delta / \mu$ and $\operatorname{Re} \zeta<1-\delta / \mu$. Noting that the distance of base $\left(\mathcal{C}_{0}\right)$ and base $(\mathcal{C})$ is less than 2 if $\mathcal{C}_{0}$ and $\mathcal{C}$ are incompatible, then we have

$$
\begin{aligned}
\sum_{\mathcal{C} ; \mathcal{C} i \mathcal{C}_{0}}|\widehat{\Psi}(\mathcal{C} ; \xi, \zeta)| e^{c^{*}(\mathcal{C})+d^{*}(\mathcal{C})} & =\sum_{\mathcal{C} ; \mathcal{C} i \mathcal{C}_{0}}|\widehat{\Psi}(\mathcal{C} ; \xi, \zeta)| \exp \left\{\sum_{\eta \in \mathcal{C}} c(\eta)+\sum_{\eta \in \mathcal{C}} d(\eta)\right\} \\
\leq & \sum_{x \in\left[\hat{l}_{0}-2, \hat{r}_{0}+2\right]} \sum_{n=1}^{\infty} \sum_{\substack{I_{1}, \ldots, I_{n} \subset[0, L] ; \cup I_{u} \ni x \\
\text { linked intervals }}} \sum_{\substack{\eta_{1}, \ldots, \eta_{n} \in \mathcal{K}_{L} ; \\
\text { base }\left(\eta_{u}\right)=I_{u}, 1 \leq u \leq n}} \\
& \prod_{u=1}^{n}\left[\widehat{\Psi}\left(\eta_{u} ; \xi, \zeta\right) e^{c\left(\eta_{u}\right)+d\left(\eta_{u}\right)}\right] \partial_{l}\left(\eta_{1}\right) \partial_{r}\left(\eta_{n}\right) \prod_{u=1}^{n-1} \partial\left(\eta_{u}, \eta_{u+1}\right) .
\end{aligned}
$$

From definitions (3.18)-(3.21) and the formula (4.35), there exists $\mu_{3}^{\prime}>0$ such that $\left|\partial_{l}\right|,\left|\partial_{r}\right|$, and $|2|$ are all bounded from above by $e^{\mu_{3}^{\prime}}$ if $\operatorname{Re} \xi<1-\delta / \mu$ and $\operatorname{Re} \zeta<1-\delta / \mu$. Therefore, from (4.36) or Lemma 4.2, we have that

$$
\begin{gathered}
\sum_{\substack{\eta_{1}, \ldots, \eta_{n} \in \mathcal{K}_{L} ; \\
\operatorname{base}\left(\eta_{u}\right)=I_{u}, 1 \leq u \leq n}} \prod_{u=1}^{n}\left[\widehat{\Psi}\left(\eta_{u} ; \xi, \zeta\right) e^{c\left(\eta_{u}\right)+d\left(\eta_{u}\right)}\right] \partial_{l}\left(\eta_{1}\right) \partial_{r}\left(\eta_{n}\right) \prod_{u=1}^{n-1} \partial\left(\eta_{u}, \eta_{u+1}\right) \\
\leq \prod_{u=1}^{n} e^{-\left(\mu_{2}-3 \mu_{1}-\mu_{3}-2 \mu_{3}^{\prime}-6\right)\left|I_{u}\right|}\left(\frac{R\left(\mu_{1}, \delta\right)^{\left|I_{u}\right|}}{1-R\left(\mu_{1}, \delta\right)}\right)^{2} .
\end{gathered}
$$


Assuming that $\mu_{2}>3 \mu_{1}+\mu_{3}+2 \mu_{3}^{\prime}+6$, then, from the inequalities (4.45) and (4.46) and if $\mu_{1}$ is large enough (by following the similar estimate procedure of (4.37) in Lemma 4.2), we obtain

$$
\begin{aligned}
\sum_{\mathcal{C} ; \mathcal{C} i \mathcal{C}_{0}}|\widehat{\Psi}(\mathcal{C} ; \xi, \zeta)| e^{e^{*}(\mathcal{C})+d^{*}(\mathcal{C})} & \leq\left(\widehat{r}_{0}-\widehat{l}_{0}+4\right) \sum_{n=1}^{\infty} \sum_{u=1}^{n} \sum_{\substack{I_{1}, \ldots, I_{n} \subset[0, L] ; \cup I_{u} \ni x \\
\text { linked intervals }}}^{n}\left(\frac{R\left(\mu_{1}, \delta\right)^{\left|I_{u}\right|}}{1-R\left(\mu_{1}, \delta\right)}\right)^{2} \\
& \leq \frac{\left(\widehat{r}_{0}-\widehat{l}_{0}+4\right) R\left(\mu_{1}, \delta\right)^{2}}{\left(1-R\left(\mu_{1}, \delta\right)\right)^{2}\left(1-R\left(\mu_{1}, \delta\right)^{2}\right)^{2}} \sum_{n=1}^{\infty} n\left(\frac{2 R\left(\mu_{1}, \delta\right)^{2}}{\left(1-R\left(\mu_{1}, \delta\right)^{2}\right)^{2}}\right)^{n-1} \\
& \leq \frac{\left(\widehat{r}_{0}-\widehat{l}_{0}+4\right)}{2} \\
& \leq c^{*}\left(\mathcal{C}_{0}\right) .
\end{aligned}
$$

Since $\sum_{\eta \in \mathcal{C}_{0}}|\operatorname{base}(\eta)| \geq \max \left\{(2 / 3)\left[\operatorname{base}\left(\mathcal{C}_{0}\right)\right], 1\right\}$ and, by the definition $\mathcal{C}^{*}(\mathcal{C})=\sum_{\eta \in \mathcal{C}} \mathcal{C}(\eta)$ and $c(\eta)=3 \mid$ base $(\eta) \mid$ (which is given in Lemma 4.2), the above last inequality holds.

According to the above inequality (4.44), we apply the general theory of cluster expansion for the partition function to display the following results; for the details, see [19]. Let $D_{f}(C D)$ be the collection of all finite subsets of $C D$, so that there exists a function

$$
\widehat{\Psi}^{T}: p_{f}(C D) \times \mathbb{C}^{2} \longrightarrow \mathbb{C}^{2}
$$

such that $\hat{\Psi}^{T}$ is analytic for $\operatorname{Re} \xi<1-\delta / \mu$ and $\operatorname{Re} \zeta<1-\delta / \mu$, and it satisfies

$$
\begin{gathered}
\sum_{\substack{\mathcal{C}_{1}, \ldots, \mathcal{C}_{z} \in \mathcal{C} \mathcal{D}_{L} ; \\
\text { compatible }}} \prod_{i=1}^{z} \widehat{\Psi}\left(\mathcal{C}_{i} ; \xi, \zeta\right)=\exp \left\{\sum_{\Delta \in \mathcal{D}_{f}\left(\mathcal{C} D_{L}\right)} \widehat{\Psi}^{T}(\Delta ; \xi, \zeta)\right\}, \\
\sum_{\Delta i \mathcal{C}_{0}}\left|\widehat{\Psi}^{T}(\Delta ; \xi, \zeta)\right| e^{d^{*}(\Delta)} \leq c^{*}\left(\mathcal{C}_{0}\right),
\end{gathered}
$$

where $d^{*}(\Delta)=\sum_{\mathcal{C} \in \Delta} d^{*}(\mathcal{C})$. If $\Delta$ is decomposed into two disjoint subsets $\Delta_{1}$ and $\Delta_{2}$, such that, for each pair $\mathcal{C}_{1} \in \Delta_{1}, \mathcal{C}_{2} \in \Delta_{2},\left\{\mathcal{C}_{1}, \mathcal{C}_{2}\right\}$ are compatible, then $\widehat{\Psi}^{T}(\Delta ; \xi, \zeta)=0$. We call $\Delta \in P_{f}\left(C P_{L}\right)$ a cluster if there are no such decomposition $\Delta=\Delta_{1} \cup \Delta_{2}$. Note that $\widehat{\Psi}^{T}(\Delta ; \xi, \zeta)$ is invariant under horizontal translation of $\Delta$. For $\Delta \in D_{f}(\mathcal{C} D)$, we set base $(\Delta)=\cup_{\mathcal{C} \in \Delta}$ base $(\mathcal{C})$. Then, by the cluster expansion theory of [19] and (4.49), we have that the limit

$$
\begin{aligned}
\widehat{\varphi}(\xi, \zeta) & =\lim _{L \rightarrow \infty} \frac{1}{L} \ln \sum_{\substack{\mathcal{C}_{1}, \ldots, \mathcal{C}_{z} \in \mathcal{C} p_{L} ; \\
\text { compatible }}} \prod_{i=1}^{z} \widehat{\Psi}\left(\mathcal{C}_{i} ; \xi, \zeta\right) \\
& =\sum_{\substack{\Delta \in p_{f}(\mathcal{C} p) ;[\text { base }(\Delta)]=[0, k] \\
\text { for some } k \geq 0}} \widehat{\Psi}^{T}(\Delta ; \xi, \zeta),
\end{aligned}
$$

exists and is analytic for $\operatorname{Re} \xi<1-\delta / \mu, \operatorname{Re} \zeta<1-\delta / \mu$ if $\mu>\mu_{2}$.

From (4.40) and (4.50), we complete the proof of Theorem 2.1. 


\section{Acknowledgments}

The authors were supported in part by National Natural Science Foundation of China Grant nos. 70471001, 70771006, and 10971010, and by the BJTU Foundation Grant no. S11M00010.

\section{References}

[1] J. Bricmont, J. L. Lebowitz, C. E. Pfister, and E. Olivieri, “Nontranslation-invariant Gibbs states with coexisting phases. I. Existence of sharp interface for Widom-Rowlinson type lattice models in three dimensions," Communications in Mathematical Physics, vol. 66, no. 1, pp. 1-20, 1979.

[2] Y. Higuchi, J. Murai, and J. Wang, "The Dobrushin-Hryniv theory for the two-dimensional lattice Widom-Rowlinson model," in Stochastic Analysis on Large Scale Interacting Systems, vol. 39 of Advanced Studies in Pure Mathematics, pp. 233-281, 2004.

[3] J. Wang, "The statistical properties of the interfaces for the lattice Widom-Rowlinson model," Applied Mathematics Letters, vol. 19, no. 3, pp. 223-228, 2006.

[4] J. Wang and S. Deng, "Fluctuations of interface statistical physics models applied to a stock market model," Nonlinear Analysis, vol. 9, no. 2, pp. 718-723, 2008.

[5] M. F. Chen, From Markov Chains to Non-Equilibrium Particle Systems, World Scientific Publishing, River Edge, NJ, USA, 2nd edition, 2004.

[6] R. Dobrushin and O. Hryniv, "Fluctuations of the phase boundary in the 2D Ising ferromagnet," Communications in Mathematical Physics, vol. 189, no. 2, pp. 395-445, 1997.

[7] R. Dobrushin, R. Kotecky, and S. Shlosman, Wullf Construction. A Global Shape from Local Interaction, vol. 104, American Mathematical Society, Providence, RI, USA, 1992.

[8] R. S. Ellis, Entropy, Large Deviations, and Statistical Mechanics, vol. 271, Springer, New York, NY, USA, 1985.

[9] Y. Higuchi and N. Yoshida, "Slow relaxation of stochastic Ising models with random and nonrandom boundary conditions," in New Trends in Stochastic Analysis, K. D. Elworthy, S. kusuoka, and I. Shigekawa, Eds., pp. 153-167, World Scientific Publishing, River Edge, NJ, USA, 1997.

[10] T. M. Liggett, Interacting Particle Systems, vol. 276, Springer, New York, NY, USA, 1985.

[11] J. Wang, "The stochastic Ising model with the mixed boundary conditions," Boundary Value Problems, vol. 2009, Article ID 571950, 17 pages, 2009.

[12] J. Wang, "The estimates of correlations in two-dimensional Ising model," Physica A, vol. 388, no. 5, pp. 565-573, 2009.

[13] J. Wang, Q. Y. Wang, and J. G. Shao, "Fluctuations of stock price model by statistical physics systems," Mathematical and Computer Modelling, vol. 51, no. 5-6, pp. 431-440, 2010.

[14] J. Bricmont and J. L. Lebowitz, "Wetting in Potts and Blume-Capel models," Journal of Statistical Physics, vol. 46, no. 5-6, pp. 1015-1029, 1987.

[15] J. De Coninck, A. Messager, S. Miracle-Solé, and J. Ruiz, "A study of perfect wetting for Potts and Blume-Capel models with correlation inequalities," Journal of Statistical Physics, vol. 52, no. 1-2, pp. 45-60, 1988.

[16] B. Derrida and M. Schick, "Interfacial wetting in the q-state Potts model," Journal of Physics A, vol. 19, no. 8, pp. 1439-1448, 1986.

[17] A. Messager, S. Miracle-Sole, J. Ruiz, and S. Shlosman, "Interfaces in the Potts model. II. Antonov's rule and rigidity of the order disorder interface," Communications in Mathematical Physics, vol. 140, no. 2, pp. 275-290, 1991.

[18] J. De Coninck, R. Koteck, L. Laanait, and J. Ruiz, "SOS approximants for Potts crystal shapes," Physica A, vol. 189, no. 3-4, pp. 616-634, 1992.

[19] R. Kotecky and D. Preiss, "Cluster expansion for abstract polymer models," Communications in Mathematical Physics, vol. 103, no. 3, pp. 491-498, 1986. 


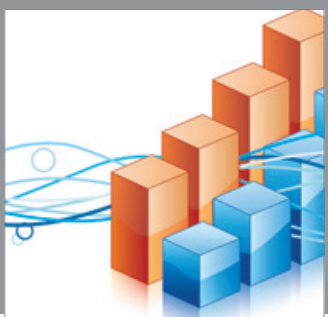

Advances in

Operations Research

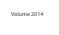

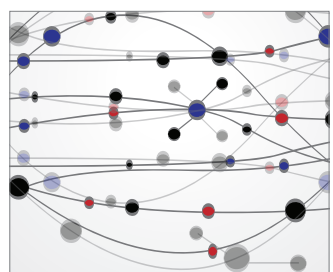

\section{The Scientific} World Journal
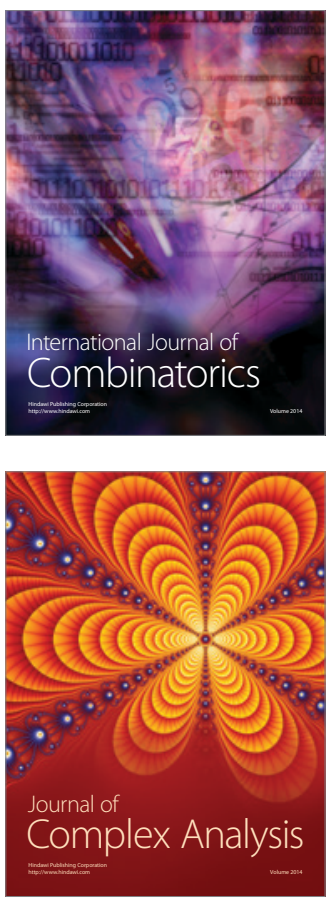

International Journal of

Mathematics and

Mathematical

Sciences
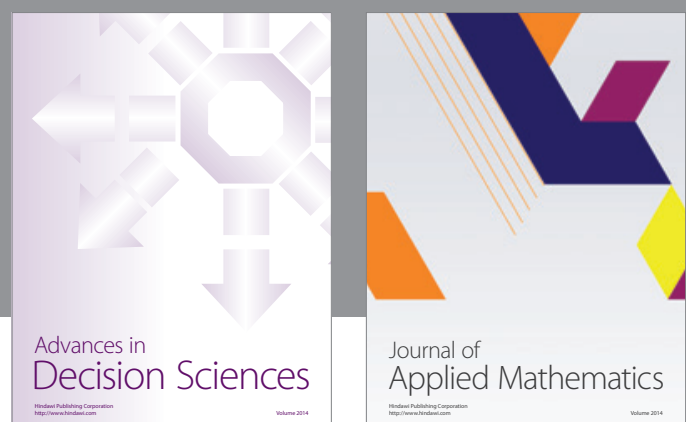

Journal of

Applied Mathematics
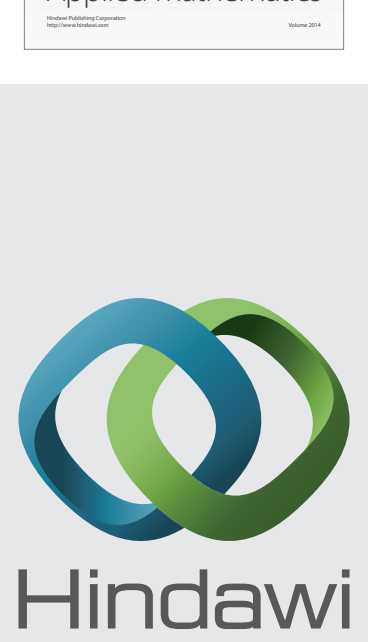

Submit your manuscripts at http://www.hindawi.com
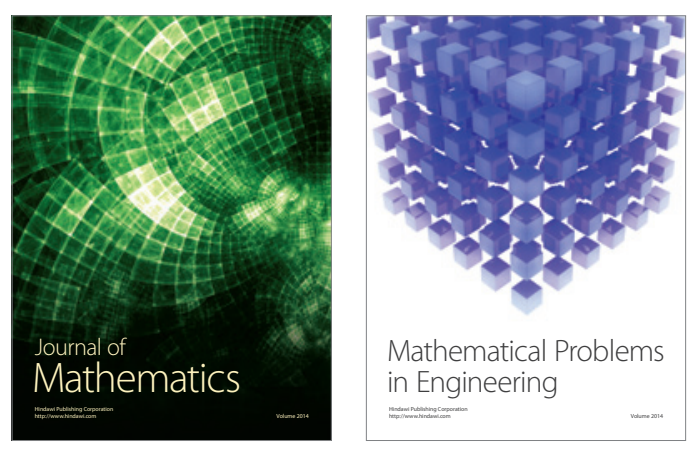

Mathematical Problems in Engineering
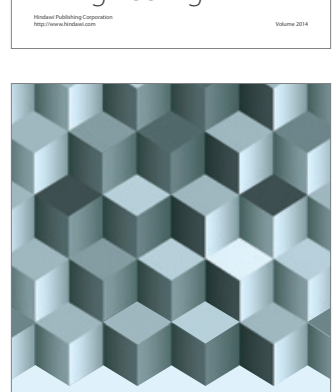

Journal of

Function Spaces
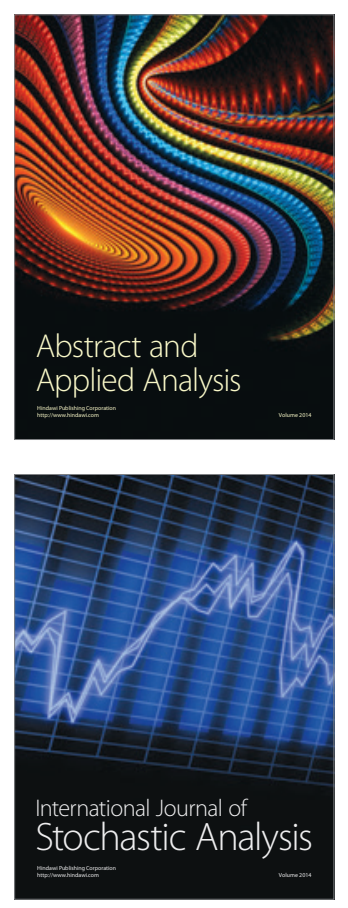

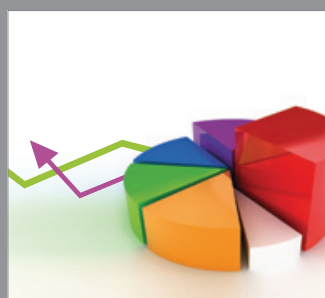

ournal of

Probability and Statistics

Promensencen
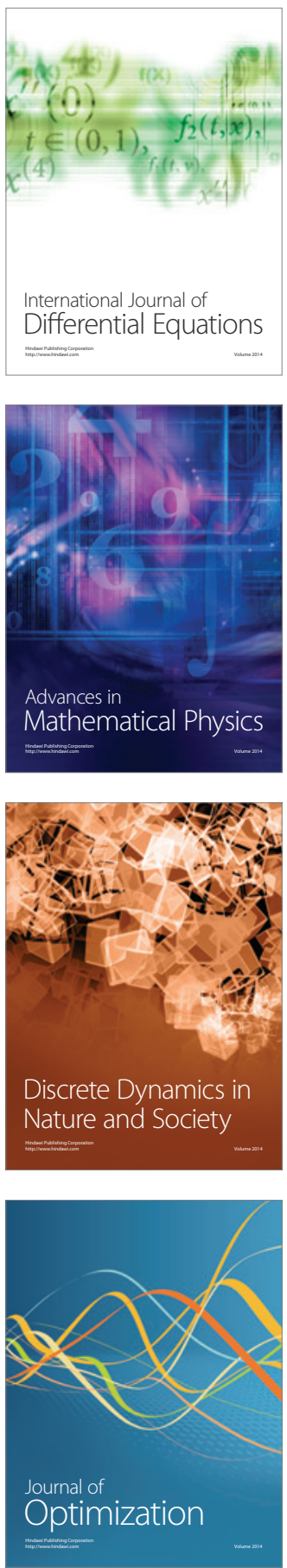